\title{
LONG-TIMESCALE BEHAVIOR OF THE BLAZHKO EFFECT FROM RECTIFIED KEPLER DATA
}

\author{
J. M. BenKő ${ }^{1}$, E. Plachy ${ }^{1,2}$, R. Szabó ${ }^{1}$, L. MolnáR ${ }^{1}$, And Z. Kolláth ${ }^{1,2}$ \\ ${ }^{1}$ Konkoly Observatory, MTA CSFK, Konkoly Thege Miklós út 15-17., H-1121 Budapest, Hungary; benko@konkoly.hu \\ ${ }^{2}$ Institute of Mathematics and Physics, Savaria Campus, University of West Hungary H-9700 Szombathely, Károlyi Gáspár tér 4, Hungary \\ Received 2014 April 22; accepted 2014 June 20; published 2014 July 23
}

\begin{abstract}
In order to benefit from the four year unprecedented precision of the Kepler data, we extracted light curves from the pixel photometric data of the Kepler space telescope for 15 Blazhko RR Lyrae stars. To collect all the flux from a given target as accurately as possible, we defined tailor-made apertures for each star and quarter. In some cases, the aperture finding process yielded sub-optimal results, because some flux have been lost even if the aperture contains all available pixels around the star. This fact stresses the importance of those methods that rely on the whole light curve instead of focusing on the extrema $(O-C$ diagrams and other amplitude independent methods). We carried out detailed Fourier analysis of the light curves and the amplitude independent $O-C$ diagram. We found $12(80 \%)$ multiperiodically modulated stars in our sample. This ratio is much higher than previously found. Resonant coupling between radial modes, a recent theory for explaining the Blazhko effect, allows single, multiperiodic, or even chaotic modulations. Among the stars with two modulations, we found three stars (V355 Lyr, V366 Lyr, and V450 Lyr) where one of the periods dominates in amplitude modulation, but the other period has a larger frequency modulation amplitude. The ratio between the primary and secondary modulation periods is almost always very close to the ratios of small integer numbers. It may indicate the effect of undiscovered resonances. Furthermore, we detected the excitation of the second radial overtone mode $f_{2}$ for three stars where this feature was formerly unknown. Our data set comprises the longest continuous, most precise observations of Blazhko RR Lyrae stars ever published. These data are made publicly available and will be unrivaled for years to come.
\end{abstract}

Key words: space vehicles - stars: oscillations - stars: variables: RR Lyrae - techniques: photometric

Online-only material: color figures, machine-readable table

\section{INTRODUCTION}

The long and (almost) uninterrupted observations of the Kepler space telescope allow us to investigate moderate or small amplitude brightness variations with long periods. These studies generally need lots of telescope time and high precision simultaneously; therefore, space photometry is ideal for them.

Such an interesting phenomenon is the Blazhko effect (Blazhko 1907) of RR Lyrae stars, the long-period amplitude modulation and frequency modulation (hereafter AM and FM, respectively) of the observed light curves. Formerly, the effect was defined as the presence of (at least) one of these two types of modulations; however, recent investigations have always found both of them simultaneously. The typical pulsation (light variation) period of a fundamental mode pulsating RR Lyrae (RRab) star is about half a day with 0.5-1 mag amplitude, while the Blazhko modulation timescale is generally $10-1000$ times longer and its amplitude is around a few tenths of magnitudes or smaller.

These behaviors encumbered the investigation of the Blazhko effect in the past. Now, the long time coverage, high duty cycle, and precise observations of Kepler allow us to address questions such as how regular the Blazhko effect is and how frequent multiperiodic Blazhko stars are. Better knowledge of the Blazhko phenomenon is important, because the effect is frequent (the incidence of the Blazhko effect among RR Lyrae stars is high: $30 \%-50 \%$ depending on the different samples considered) and its physical origin-despite the serious efforts during the last hundred years-is still unknown.

As of today, two competitive explanations of the Blazhko effect have survived among the many previously suggested ones (for a review, see Kolenberg 2012 or Kovács 2009). Stothers' idea (Stothers 2006) explains the effect with the influence of local transient magnetic fields excited by the turbulent convection. The first quantitative tests of this idea found serious inconsistencies between the theory and observations (Smolec et al. 2011; Molnár et al. 2012a). Buchler \& Kolláth (2011) suggested a model where the modulation caused by a resonance coupling between a low order radial (typically fundamental) mode and a high order radial (the so-called strange) mode. This model is based on the amplitude equation formalism, but it has not been tested yet by hydrodynamic computations. Both of these theories can potentially predict variable Blazhko cycles. In the first case the variation could be quasi-periodic with a stochastic nature, while in the second case it can be regular: single, multiperiodic, or chaotic.

\section{DATA}

The main characteristics of the Kepler Mission are described in Koch et al. (2010) and Jenkins et al. (2010a, 2010b). Technical details can be found in the following handbooks: Van Cleve et al. (2009), Fanelli et al. (2011), and Jenkins et al. (2013). We summarize here only those basic facts about the instrument that proved to be important in our analysis.

The space telescope orbits the Sun and observed one fixed area continuously at the Cygnus/Lyra region. To ensure the optimal illumination for its solar cells, the equipment was rolled by $90^{\circ}$ four times a year. As a consequence, each target star's light is collected on four different CCDs according to the quarterly positions. This implies possible systematic differences among data from different quarters. When we have a look at the flux variation curves of an RR Lyrae star, the zero points and amplitudes are evidently different from quarter to quarter for most of the stars (see Figure 1 for an example). 


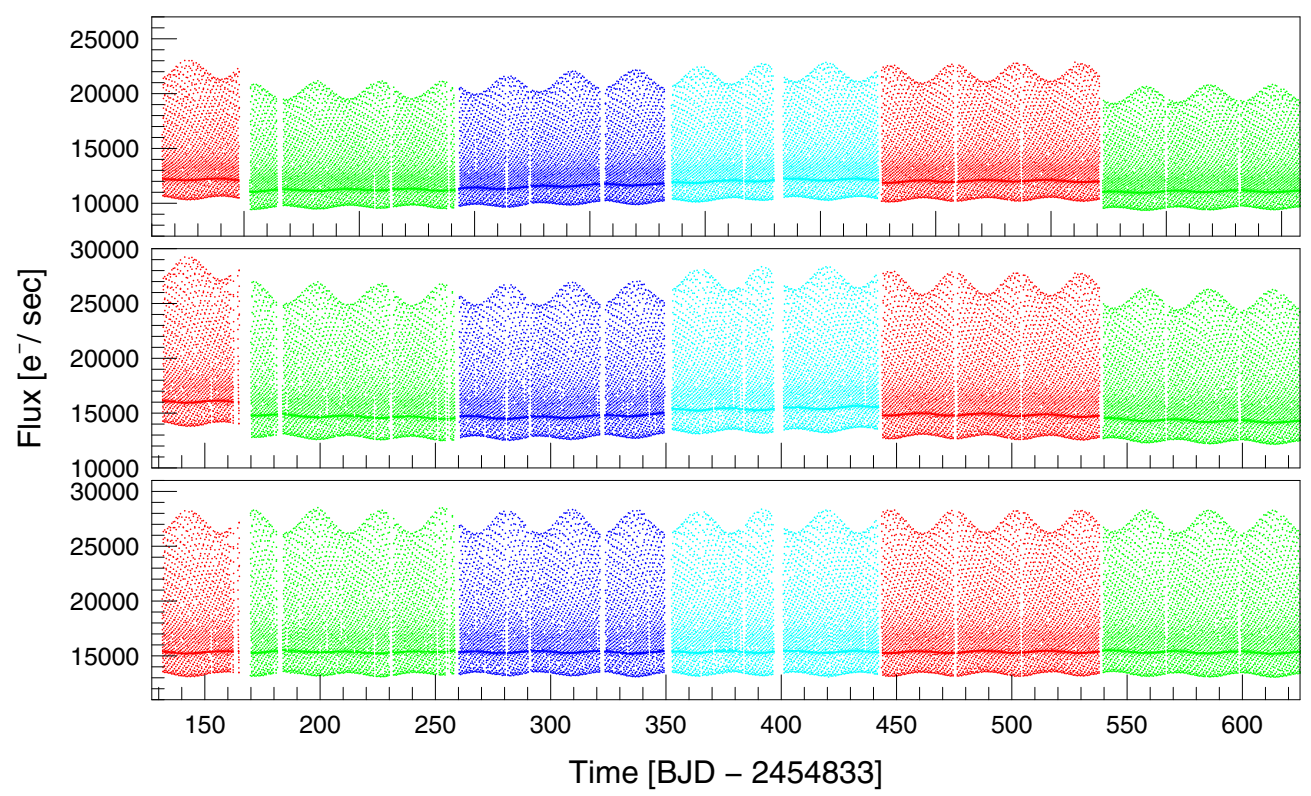

Figure 1. Top panel: "optimal aperture" flux variation curve of V783 Cyg (Kepler archive). Middle panel: the flux variation curve prepared by using the best tailor-made aperture. Bottom panel: scaled, shifted, and detrended curve. (For better visibility, only the first six quarters are plotted.)

(A color version of this figure is available in the online journal.)

Due to the limited telemetrical capacity, only small areas around each selected target star were downloaded. We will refer to these areas in this paper as "stamps." Within these stamps, "optimal apertures" (in Kepler jargon) were fitted from the 1024 predefined ones for each star and quarter separately. The photometry done on these apertures defines the Kepler flux variation curves. These apertures are, however, optimal only if the light variation of the target is less than about a tenth of a magnitude (Fanelli et al. 2011). Since the total amplitude of pulsation for Kepler RR Lyrae stars is between 0.47 and 1.1 mag (Nemec et al. 2013, hereafter N13), these predefined apertures are not optimal anymore: a significant fraction of the flux flows out of these apertures. This effect adds to the fact that even the apertures may differ quarterly for a given star, explaining most of the differences between the amplitudes and average fluxes pertaining to different quarters.

\subsection{The Sample}

We assembled our Blazhko star sample in the Kepler field as follows. The sample of Benkô et al. (2010) contains 14 stars. One of them-V349 Lyr (KIC 7176080) - proved to be a nonmodulated star suggested by Nemec et al. (2011). This finding has been confirmed by the present work by checking its pixel photometric data. We also include three additional stars that were analyzed by N13. The extremely small Blazhko effect of V838 Cyg (KIC 10789273) was revealed by N13, while the (Blazhko) RR Lyrae nature of KIC 7257008 and KIC 9973633 was discovered by the ASAS survey (Pojmanski 1997, 2002; R. Szabó et al., in preparation) when the Kepler measurements were in progress. That is the reason why we have data for these two targets from Q10 only. The other 13 stars were preselected by the Kepler Asteroseimic Science Consortium ${ }^{3}$ and were observed during the whole mission. N13 shortly noted two additional Blazhko candidates; however, both of those stars are faint and merging with neighboring bright sources. Because of the serious problems concerning the separation of their

\footnotetext{
3 http://astro.phys.au.dk/KASC/
}

signals from their close companions, we omitted them from our investigations. (They will be discussed in a forthcoming paper.) RR Lyr itself is also in the Kepler field, but its image is always saturated. Therefore, recovering its original signal requires extra caution and special techniques (e.g., custom apertures). Many successful efforts have been made in this direction (Kolenberg et al. 2010, 2011; Molnár et al. 2012b). We will only refer to those results for RR Lyr. Our final sample consists of fifteen Blazhko stars.

The exposition time of the Kepler camera is $6.02 \mathrm{~s}$ with $0.52 \mathrm{~s}$ readout time. The long-cadence (LC) data result from 270 exposures co-added with a total $1766 \mathrm{~s}$ integration time. Since we concentrate on long-period effects, we generally used these LC data only, especially since the time spans of short cadence (SC) data (nine frames codded $58.85 \mathrm{~s}$ ) are usually no more than one single quarter.

The commissioning phase data (Q0) between 2009 May 2 to 11 (9.7 days) included only one Blazhko star: KIC 11125706. The observations of other targets began with Q1 on 2009 May 13. Here we analyzed LC data to the end of the last full quarter (Q16, until 2013 April 8). The total length of the data covers 3.9 yr. The CCD module No. 3 failed during Q4 (on 2010 January 12), and the targets located on this module have quarterlong gaps in their time series data. Six stars of our 15-element sample suffer from this defect (see Table 3 and Figure 4). The combined number of data points for a given star is between 19249 (KIC 9973633) and 61351 (V783 Cyg), and the typical value is about $50,000-60,000$. Data are public and can be downloaded from the MAST Web site. ${ }^{4}$

\subsection{Data Processing}

In this subsection, we summarize the main steps that were performed before our analysis. The Kepler data available for each source are in two forms: (1) as a photometric time series, flux variation curves (flux versus time) prepared from the predefined optimal apertures, and (2) as an image time series

4 http://archive.stsci.edu/kepler/ 

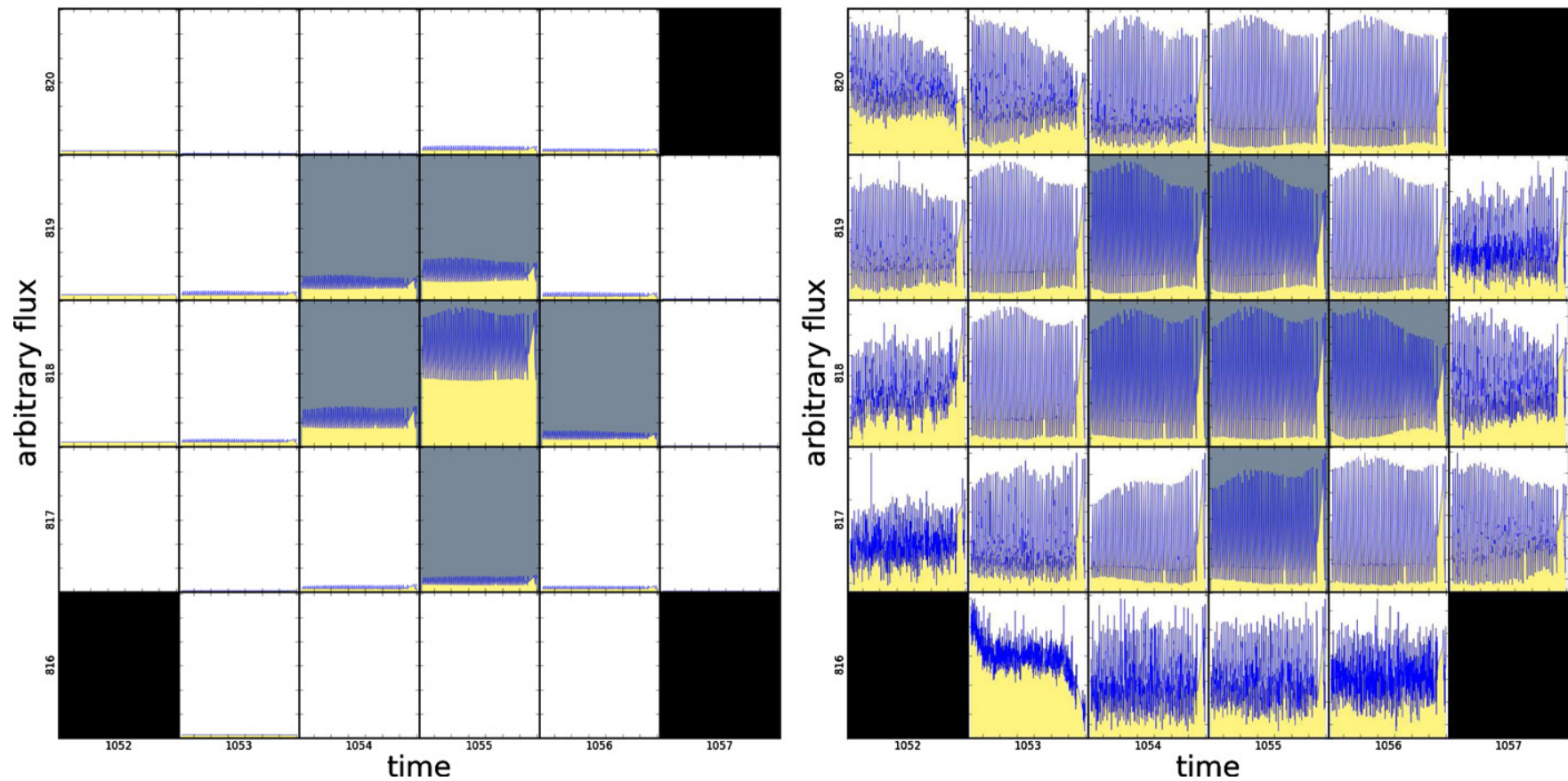

Figure 2. Construction of the tailor-made apertures. The entire pixel mask around V783 Cyg (KIC 5559631) in the first quarter is shown. Gray pixels: elements of the "optimal apertures." White pixels: all other downloaded pixels ("stamp"). We plotted individually the Q1 time series of each pixel. On the left-hand side, all pixel light curves are scaled between zero and the maximum flux. On the right-hand side, all pixel light curves are scaled individually. We collected all those pixels that show the signal of the star and omitted those that include noise or background sources only.

(A color version of this figure is available in the online journal.)

(image of the stamp versus time). The latter data sets are often referred to as "pixel data." For the above mentioned reasons, we used these pixel data. After we downloaded them from the MAST Web page PyKE, ${ }^{5}$ routines provided by the Kepler Guest Observer Office were used to extract the flux variation curves for each pixel in the stamps of a given star. Since the pixel files before file version 5.0 have a time stamp error, we corrected it by using the PyKE keptimefix tool.

We investigated the flux variation curves of all individual pixels separately. We illustrate this process in Figure 2. Here the stamp of Q1 around the star V783 Cyg (KIC 5559631) is plotted on two different scales. The gray pixels symbolize the elements of the predefined "optimal" aperture. We plotted the Q1 time series of each pixel individually. On the left panel all pixel flux variation curves are scaled between zero and the maximum flux attained by the brightest pixel in the stamp. This option shows the relative contribution to the archived flux variation curve by each pixel. The total flux of the star obviously comes from only a few pixels. On the right panel all individual pixel flux variation curves are scaled separately between their minima and maxima to ensure the largest dynamic range for each pixel. This map reveals that there is some flux from outside of the original ("optimal") aperture.

Aiming for apertures for each star and quarter separately that include the total flux, we built the "tailor-made" apertures in the following way. If the flux variation curve of a pixel showed the signal of the given variable star-that is, the main pulsation period is detectable $\left(A\left(f_{0}\right) \sim 3 \sigma\right)$ in the Fourier spectrum-we added the pixel in question to our tailor-made aperture, but otherwise we dropped it. By summing up the flux of all pixels in the tailor-made apertures, we obtained raw flux variation curves.

\footnotetext{
5 http://keplergo.arc.nasa.gov/PyKE.shtml
}

At first sight, these time series and the Kepler flux variation curves do not differ too much (see Figure 1 for an example). Nevertheless, the difference between the flux values of the archived ("optimal" aperture) data and our ("tailor-made" aperture) fluxes is considerable: about $1 \%-5 \%$. The exact value differs from star to star and from quarter to quarter. We note that such a comparison requires shifting the quarters pairwise to a common zero point.

The main differences between the archived data and our flux variation curves are that (1) the total pulsation amplitudes $A_{i}^{\mathrm{T}}(n)$ increase for all quarters $\left(A_{i}^{\prime \mathrm{T}}(n)>A_{i}^{\mathrm{T}}(n)\right.$; Figure 3$)$, indicating the flux loss in the archived data. Here the amplitudes $A_{i}^{\mathrm{T}}(n), A_{i}^{\prime \mathrm{T}}(n)$ are the total pulsation amplitude (maximal flux - minimal flux) of the $n$th pulsation cycle in the $i$ th quarter $i, n=1,2, \ldots$ for optimal and tailor-made aperture data, respectively. (The superscript $\mathrm{T}$ stands for the word "total.") (2) The internal trends within quarters (see arrows in Figure 3) decrease, suggesting that these trends originate from the small drift and differential velocity aberration of the telescope; and (3) the difference of the total pulsation amplitudes between the consecutive quarters $\Delta A_{i, i+1}^{\mathrm{T}}=\left|A_{i}^{\mathrm{T}}(l)-A_{i+1}^{\mathrm{T}}(1)\right|$ decrease: $\Delta A_{i, i+1}^{\mathrm{T}}>\Delta A_{i, i+1}^{\prime \mathrm{T}}$ (the index $l$ denotes the last pulsation cycle in the $i$ th quarter). In an optimal case-if the tailor-made apertures capture all the flux - these total pulsation amplitude differences practically disappear and only zero point shifts would remain between quarters.

Initially, we hoped that we could define tailor-made apertures for all stars that include the total flux, i.e., the different quarters can be joined smoothly by simple zero point shifts. We have found such apertures for nine Blazhko stars only. Six of our stars, however, show total pulsation amplitude differences between quarters for all possible apertures. (For the list of the individual stars, see Table 3.) In these cases the downloaded stamps seemed to be too small. The right panel in Figure 2 demonstrates such 

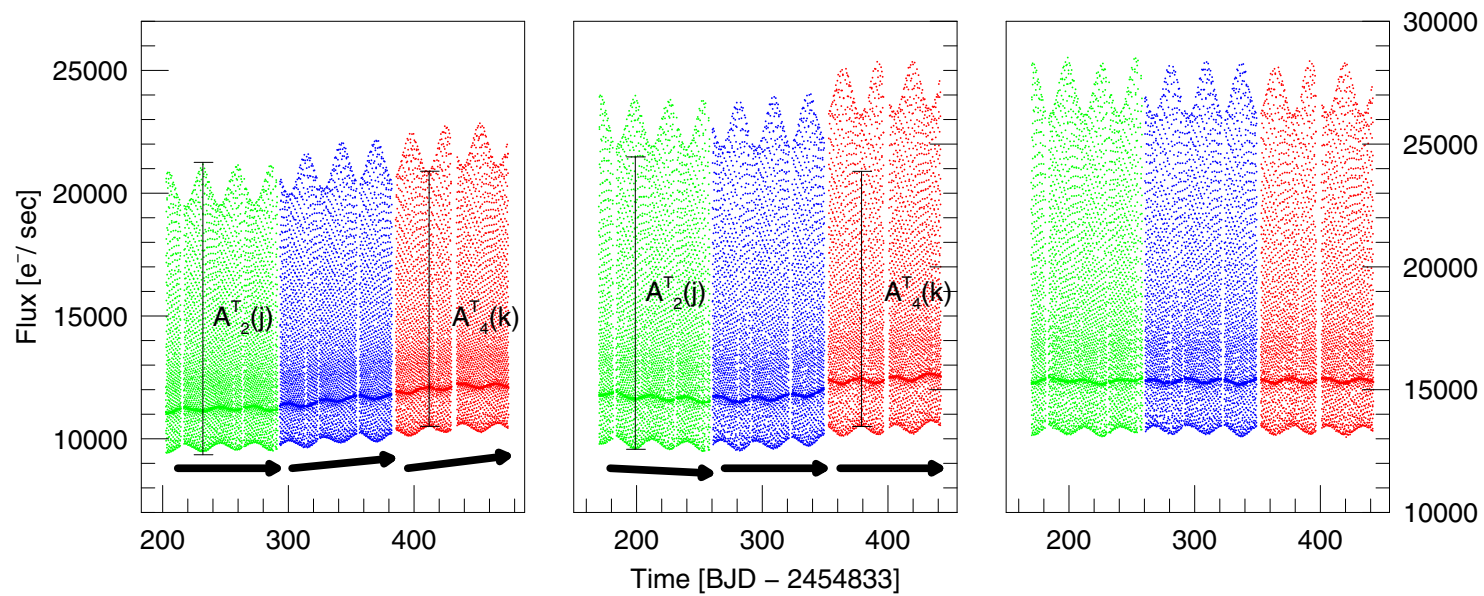

Figure 3. Flux variation curves from data processing. The figure shows curves of V783 Cyg from Q2 to Q4. From left to the right: the archived Kepler data, fluxes extracted from the best tailor-made apertures, and final rectified data, respectively. The arrows illustrate the internal trends. $A_{2}^{\mathrm{T}}(j)$ and $A_{4}^{\mathrm{T}}(k)$ are the total pulsation amplitudes of the archived Kepler Q2 and Q4 data at the $j$ th and $k$ th pulsation cycles, respectively. These amplitudes are also shown in the tailor-made data plot for comparison.

(A color version of this figure is available in the online journal.)

Table 1

Sample from a Rectified Data File

\begin{tabular}{lccccrr}
\hline \hline No & $\begin{array}{c}\text { Time } \\
(\text { BJD-2454833) }\end{array}$ & $\begin{array}{c}\text { Flux } \\
\left(e^{-} \mathrm{s}^{-1}\right)\end{array}$ & $\begin{array}{c}\text { Zero Point Offset } \\
\left(e^{-} \mathrm{s}^{-1}\right)\end{array}$ & Scaling Factor & $\begin{array}{c}\text { Corrected Flux } \\
\left(e^{-} \mathrm{s}^{-1}\right)\end{array}$ & $\begin{array}{c}\text { Corrected } K_{\mathrm{p}} \\
(\mathrm{mag})\end{array}$ \\
\hline 1 & 131.5123241 & 5322.6 & -400.00 & 1.000 & 5402.03436789 & 0.39793763 \\
2 & 131.5327588 & 5393.9 & -400.00 & 1.000 & 5473.33140201 & 0.38370163 \\
3 & 131.5531934 & 5496.7 & -400.00 & 1.000 & 5576.12843615 & 0.36349907 \\
4 & 131.5736279 & 5498.1 & -400.00 & 1.000 & 5577.52547030 & 0.36322708 \\
5 & 131.5940625 & 5488.2 & -400.00 & 1.000 & 5567.62250444 & 0.36515654 \\
6 & 131.6144972 & 5571.6 & -400.00 & 1.000 & 5651.01953856 & 0.34901397 \\
7 & 131.6349317 & 6347.2 & -400.00 & 1.000 & 6426.61657271 & 0.20937502 \\
8 & 131.6553663 & 8478.6 & -400.00 & 1.000 & 8558.01360685 & -0.10160144 \\
9 & 131.6758010 & 14437.0 & -400.00 & 1.000 & 14516.41064097 & -0.67531712 \\
10 & 131.6962356 & 15817.5 & -400.00 & 1.000 & 15896.90767511 & -0.77395064 \\
$\ldots$ & $\ldots$ & $\ldots$ & $\ldots$ & $\ldots$ & $\ldots$ & $\ldots$ \\
\hline
\end{tabular}

Notes. The first 10 data lines from the file of V2178 Cyg (kplr003864443. tailor-made. dat). The columns contain serial numbers, baricentric Julian dates, flux extracted from the tailor-made aperture, zero point offsets, scaling factors $(1.0=$ no scaling $)$, stitched (shifted, scaled, and trend filtered) flux, and their transformation into the $K_{\mathrm{p}}$ magnitude scale, respectively. See the text for the details.

(This table is available in its entirety in a machine-readable form in the online journal. A portion is shown here for guidance regarding its form and content.)

a situation well: the top pixel row and the right-most column contain the signal of the variable star while, for example, the bottom row does not.

How can we correct the flux variation curve of such stars? Simple zero point shifts do not result in continuous light curves; however, we must assume that the light curve of an RR Lyrae star is continuous and smooth. To this end, we have to scale the flux values for properly joining the quarters. Since at the beginning of the mission Q4 was the most stable quarter (see Figure 10 in Jenkins et al. 2013), we chose its fluxes as a reference for all stars. We defined the scaling factor and zero point offset pairs for each quarter separately so that the transformed flux values of the quarters can be stitched smoothly. These transformations are neither exact nor unique. An additional trouble arises when one quarter of the data is missing. In those cases, since the stamps of a star were generally fixed for the identical telescope rolls (viz. settings for $\mathrm{Q} 1=\mathrm{Q} 5=\mathrm{Q} 9, \ldots ; \mathrm{Q} 2=\mathrm{Q} 6=\mathrm{Q} 10, \ldots$ etc.), we used the scaling factor and zero point offset of the previous quarter at the same telescope positions: e.g., if Q8 data are missing, we use the parameters of Q5 for Q9. It must be kept in mind that this procedure may influence the final result, especially when we investigate amplitude changes.
The flux values of each quarter were (1) shifted with zero point offsets and (2) multiplied by scaling factors. Finally, (3) the eventual long-timescale trends were removed from the flux variation curves by a trend filtering algorithm prepared for CoRoT RR Lyrae data, ${ }^{6}$ and (4) fluxes were transformed into a magnitude scale, where the averaged magnitude of each star was set to zero. We have to emphasize that due to the logarithmic nature of the magnitude scale, all corrections and transformations should be performed on the flux data. The measured fluxes fell between 1190 and 350,500 $e^{-} \mathrm{s}^{-1}$, which yield the estimated errors of $7.2 \times 10^{-4}$ and $4.2 \times 10^{-5} \mathrm{mag}$ for an individual data point, respectively. Corrected time series data on the tailor-made apertures are available both in flux and magnitude scales in electronic format. ${ }^{7}$ The fluxes extracted from the tailor-made aperture without trend filtering, applied scaling factors, and offset values are also given in the data set (for a sample, see Table 1).

\footnotetext{
6 http://www.konkoly.hu/HAG/Science/index.html

7 See the following Web page: http://www.konkoly.hu/KIK/data.html.
} 


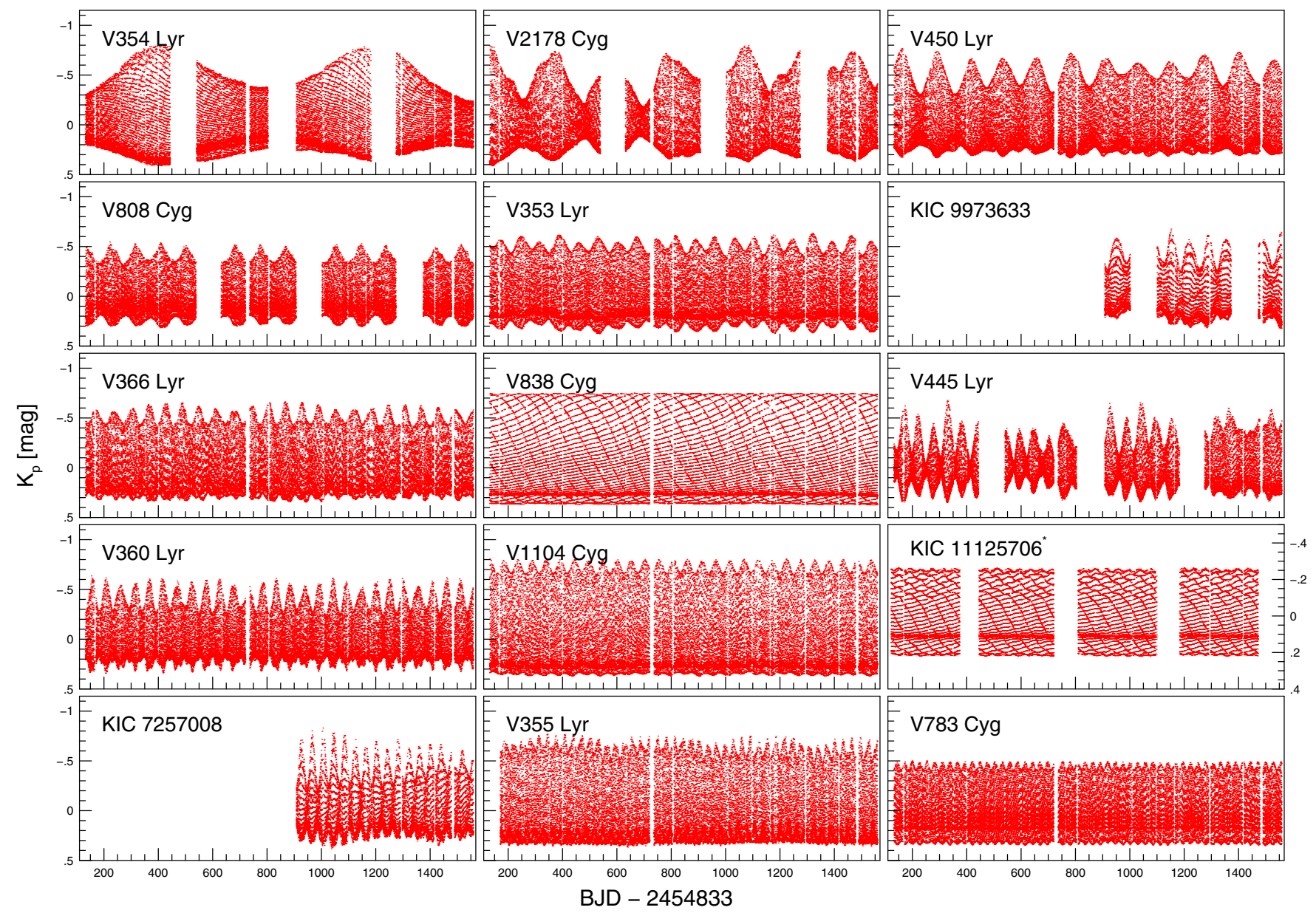

Figure 4. Gallery of Kepler Blazhko stars. The figure shows the complete light curves of fifteen stars observed with long-cadence (LC) sampling during the periods Q0 through Q16. The light curves are ordered by the primary Blazhko period from the longest (top left) to the shortest (bottom right) ones. *: for better visibility the scale of KIC 11125706 is increased by a factor of 1.5 .

(A color version of this figure is available in the online journal.)

\section{ANALYSIS AND RESULTS}

\subsection{General Overview}

In the course of this study, we mainly used two methods. One of them is the Fourier analysis of the light curves that was preconditioned by the described process and shown in Figure 4 . The second method is the analysis of $O-C$ (observed minus calculated) diagrams. In some cases, other tools were used as well. These are described later when we discuss the relevant objects.

This paper uses the following notation conventions: numbers in the lower indices denote the radial pulsation orders (viz. 0 = fundamental, $1=$ first overtone modes, etc.). Lower indices B and S indicate the primary (Blazhko) and secondary modulations, respectively. Upper indices denote the detected frequencies before identification. Throughout this paper the numerical values (frequencies, amplitudes, etc.) are written with the significant number of digits plus one digit.

\subsubsection{Fourier Analysis of the Light Curves}

The software packages MuFrAN (Kolláth 1990) and PERIOD04 (Lenz \& Breger 2005) were used for the Fourier analysis. These program packages - together with SIGSPEC (Reegen 2007) - were tested for Kepler Blazhko stars in the past (Benkô et al. 2010). Since all of them provided similar spectra with the same frequencies, amplitudes, and phases, we can use the one that fits our purposes the best. In this work, our primary tool was MuFrAn, but, for example, the frequency error or signalto-noise ratio $(\mathrm{S} / \mathrm{N})$ was determined by PERIOD04.

Here we describe the general features of our Fourier analysis. For an illustration, see Figure 5. The highest peaks in the Fourier spectra are always the main pulsation frequencies $\left(f_{0}\right)$ and their harmonics $\left(k f_{0}\right.$, where $k=1,2, \ldots$; see panel (A) in Figure 5). The Nyquist frequency for the Kepler LC data is $f_{\mathrm{N}}=$ $24.46 \mathrm{day}^{-1}$. Up to this limit frequency, we detected 9-15 significant harmonics depending on the pulsation frequency.

When we pre-whiten the data with these frequencies, we get Fourier spectra dominated by the side peaks (Figure 5(B)). The harmonics (including the main frequency) are surrounded by the side peaks caused by the Blazhko modulation $\left(k f_{0} \pm l f_{\mathrm{B}}\right.$, where $k, l=1,2, \ldots)$. Side peaks of triplets $(l=1)$ can always be seen (panel $\left(\mathrm{B}_{2}\right)$ in Figure 5), and in some cases higher order multiplets $(l>1)$ are also detectable (panel $\left.\left(\mathrm{B}_{4}\right)\right)$. The higher order multiplets tend to appear around the higher order harmonics, which indicates the FM (Benkô et al. 2011).

After we pre-whitened the data with a set of side frequencies, it became evident that the side peaks sometimes consist of double or even multiple peaks. If we measure the spacing between these double peaks, we find that the frequency difference corresponds to the Kepler year $\left(P_{\mathrm{K}}=372.5\right.$ days). More precisely, 

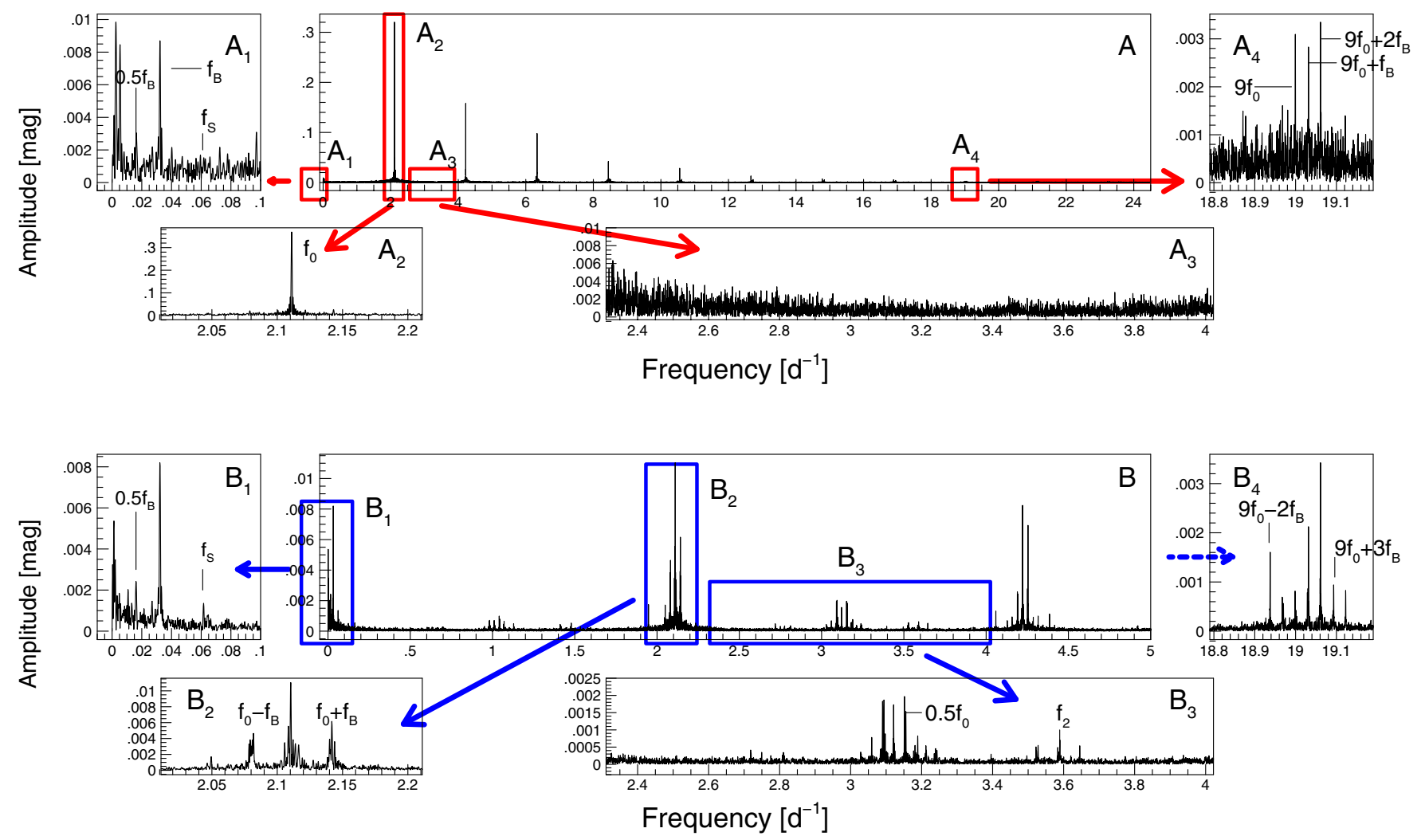

Figure 5. Schematic overview of the Fourier analysis of the light curves. Top panels (denoted by (A)): Fourier amplitude spectra. Bottom panels (denoted by (B)): spectra after we pre-whitened the data with the main pulsation frequency $f_{0}$ and its significant harmonics $k f_{0}, k=1,2, \ldots$. Panels with indexed letters are zooms of the spectra: (1) low frequency range, (2) surrounding of $f_{0}$, (3) additional frequencies between $f_{0}$ and $2 f_{0}$, and (4) high frequency range (around $9 f_{0}$ ). Colored boxes in the middle panels show the approximate positions and sizes of the small (zoomed) panels.

(A color version of this figure is available in the online journal.)

these frequencies can be described as $k f_{0} \pm f^{\prime}$, where $f^{\prime}$ is one of the following: $0.5 f_{\mathrm{K}}, f_{\mathrm{K}}, 2 f_{\mathrm{K}}$, or $4 f_{\mathrm{K}}=f_{\mathrm{Q}}$. Here $f_{\mathrm{K}}=1 / P_{\mathrm{K}}$ and $f_{\mathrm{Q}}=1 / P_{\mathrm{Q}}$, where $P_{\mathrm{Q}}$ is the characteristic length of a quarter. Thus, these frequencies are caused either by not properly eliminating problems of stitched quarters, by missing quarters, or sometimes by the instrumental amplitude variation with $P_{\mathrm{K}}$ that was recently discovered by Bányai et al. (2013).

In the low frequency ranges (panel $\left(\mathrm{B}_{1}\right)$ in Figures 5 and 8), we generally find the modulation frequencies $f_{\mathrm{B}}$, frequencies connected to the Kepler year, and some other instrumental peaks. In many cases we also find the harmonics of the Blazhko frequencies to be significant. They imply that the amplitude modulation is fairly non-sinusoidal, which is evident from the envelopes of the corresponding light curve in Figure 4. It is surprising that in many cases more than one modulation peak can be found (see also $\left(B_{1}\right)$ in Figure 5 ). These secondary modulation frequencies sometimes mimic the harmonics of the primary modulation frequencies, but the $O-C$ analysis (see Section 3.1.2) helped us to distinguish between multiple modulation and non-sinusoidal modulation. The ratios between different Blazhko frequencies are often close to small integer numbers, which may suggest a resonance at work.

Although this paper focuses on the long-timescale variations, we briefly discuss the frequency ranges between the harmonics, since the spectra after pre-whitening with the modulation frequencies and the side peaks show specific structures (see panel $\left(\mathrm{B}_{3}\right)$ in Figure 5). Four stars (V353 Lyr, V1104 Cyg, KIC 11125706, and V783 Cyg) do not show any significant peaks in these frequency ranges, but the remaining 11 stars do (Figure 6 ).
Three well-separated forests of peaks can be identified that appear in all stars with different combinations. The middle ones belong to the period doubling (PD) phenomenon (Kolenberg et al. 2010; Szabó et al. 2010; Kolláth et al. 2011). The half-integer frequencies (HIFs: $0.5 f_{0}, 1.5 f_{0}, \ldots$ ), their side peaks, and a number of linear combination frequencies can be detected. If we determine the frequency ratio of these HIF peaks, we find that their values frequently differ from the exact half-integer ratios. The explanation is a combination of mathematical, physical, and sampling effects (Szabó et al. 2010). The frequencies located between the HIFs and $2 f_{0}$ harmonics belong to the second radial overtone $\left(f_{2}\right)$, while peaks between $f_{0}$ and HIFs are identified as the frequency of the first radial overtone mode $\left(f_{1}\right)$. The explanation of the huge number of surrounding peaks around all three cases is mathematical: the amplitude of the additional frequencies for both the PD effect and overtone modes changes in time (see, e.g., Benkô et al. 2010; Szabó et al. 2010, 2014; Guggenberger et al. 2012). Such a variable signal results in a forest of peaks in the Fourier spectra as it was shown by Szabó et al. (2010).

\subsubsection{Analysis of the $O-C$ Diagram}

The FM part of the Blazhko effect can be separated if we study the effect in the time domain. Since the AM and FM definitely connected to each other, such an investigation shows different aspects of the same phenomenon. A practical advantage of this handling is that the time measurements are almost free of instrumental problems, contrary to the brightness measurements that were discussed in Section 2. 


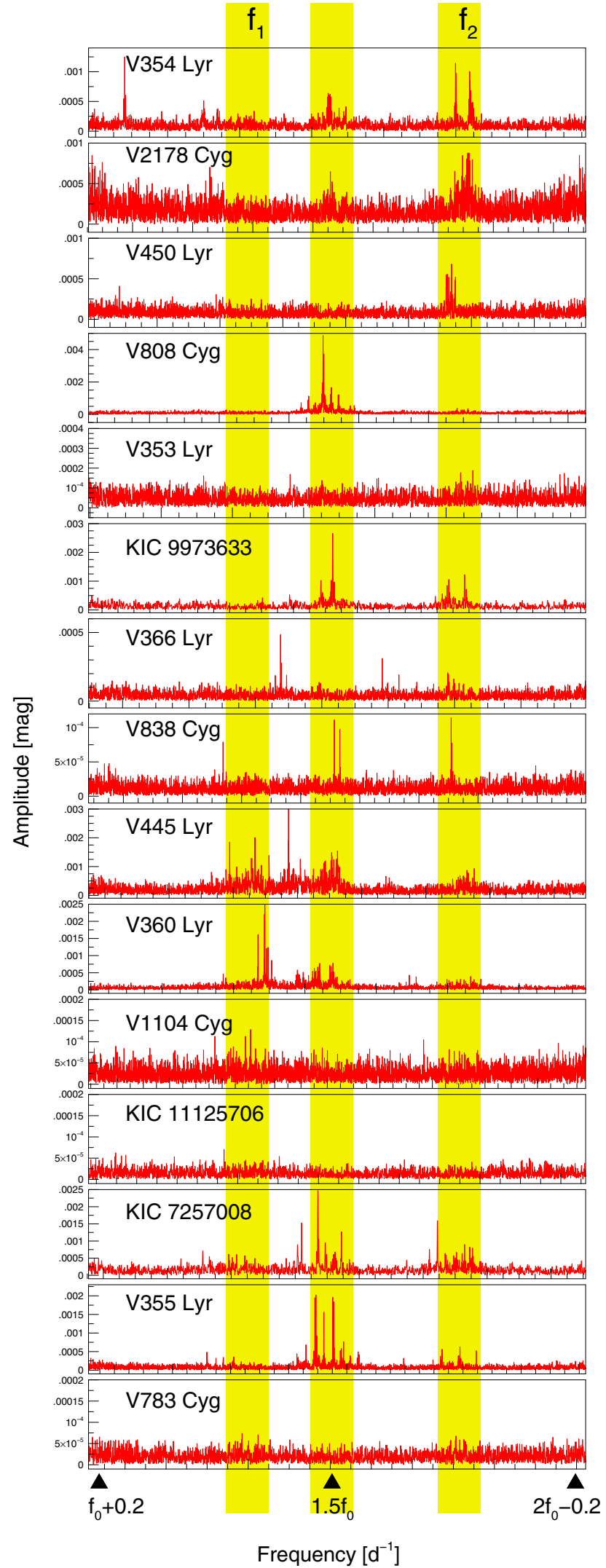

Figure 6. Structure of the additional frequencies. Here we plotted the spectra between the main frequencies and their first harmonics $\left(f_{0}+0.2<f<\right.$ $2 f_{0}-0.2$ ). All figures show residual spectra after consecutive pre-whitening steps with the harmonics and their numerous (3-10) significant side peaks. The yellow stripes indicate the expected position of the radial first overtone $\left(f_{1}\right)$, period doubling $\left(1.5 f_{0}\right)$, and radial second overtone $\left(f_{2}\right)$ frequencies, respectively.

(A color version of this figure is available in the online journal.)
There are numerous opportunities for following frequency/ period variations from the traditional $O-C$ diagram (Sterken 2005 ) to the analytic signal method (Kolláth et al. 2002), or we can transform it to phase variation as, for example, N13. We have chosen here the $O-C$ diagram analysis as a simple and clear method. Although $O-C$ diagrams were widely used for investigating RR Lyrae stars for many decades, the first diagrams that show the period variations due to the Blazhko effect were published only recently (Chadid et al. 2010; Guggenberger et al. 2012), when the continuous space-based data became available.

As defined by Sterken (2005), " $O-C$ stands for O[bserved] minus C[alculated]:... it involves the evaluation and interpretation of the discord between the measure of an observable event and its predicted or foretold value." In our case, we chose the time of maxima of the pulsation as an "observable event." For the determination of the observed maximum times (" $O$ " values), we used seventh to ninth order polynomial or spline fits around the maximum brightness of each pulsation cycle. The initial epochs $\left(E_{0}\right)$ were always the time of the first maximum for each star. The " $C$ " (calculated) maximum times were determined from these epochs and the averaged pulsation periods $\left(P_{0}\right): C=E_{0}+E P_{0}$, where $E=1,2 \ldots$ is the cycle number. Gaps in the observed light curves often resulted in interpolation errors and consequently deviant points in the constructed $O-C$ curves. We removed these points with the time string plot tool of PERIOd04. The selection criterion for the wrong points was that they deviate from the smooth fit of the curves more than $3 \sigma$, where $\sigma$ indicates the standard deviation of the fit. The obtained curves are plotted in Figure 7. The accuracy of an individual $O-C$ value is about 1 minute.

The frequency content of the $O-C$ diagrams were extracted again by Fourier analysis. The corresponding spectra are shown in Figure 8, where we compare them with the low frequency range of the light curve Fourier spectra. Generally speaking, the structures of these two types of spectra are similar, but $O-C$ spectra are more clear. Here no instrumental peaks can be detected, and in the case of multiple modulated stars the linear combination frequencies are also more numerous and significant. These linear combination frequencies demonstrate that both modulations belong to the same star (and not to a background source) and the nonlinear coupling between different modulations. It is noteworthy that the frequencies of the AM and FM are always the same within their errors.

\subsubsection{Calculated Parameters and Accuracies}

The analyzed Fourier spectra have well-defined structures, and although the spectra of the light curves contain hundreds of significant peaks, only a few of them belong to independent frequencies. These are the main pulsation frequency $f_{0}$, the Blazhko frequencies $\left(f_{\mathrm{B}}\right.$ and $\left.f_{\mathrm{S}}\right)$, and the frequencies of the excited additional radial overtone mode(s) $\left(f_{1}, f_{2}\right.$, and/or the strange mode $f_{9}$ that is responsible for the PD effect). The error estimation for both the frequencies and amplitudes were obtained by Monte Carlo simulation of PERIOD04. We note that these errors are only few percent higher than the analytic error estimations (Breger et al. 1999), because we have almost continuous, uniformly sampled data sets.

The error estimation of the main pulsation frequency yields 1.1-1.8 $\times 10^{-7} \mathrm{day}^{-1}$ for the Q1-Q16 data sets and $4 \times$ $10^{-6}$ days $^{-1}$ for the Q10-Q16 data. These translate to a $3-5 \times 10^{-8}$ day period uncertainty for the best data (short period and long observing span) and $10^{-6}$ days at worst. The Rayleigh frequency resolution is $0.0007 \mathrm{day}^{-1}$ for the Q1-Q16 data sets 


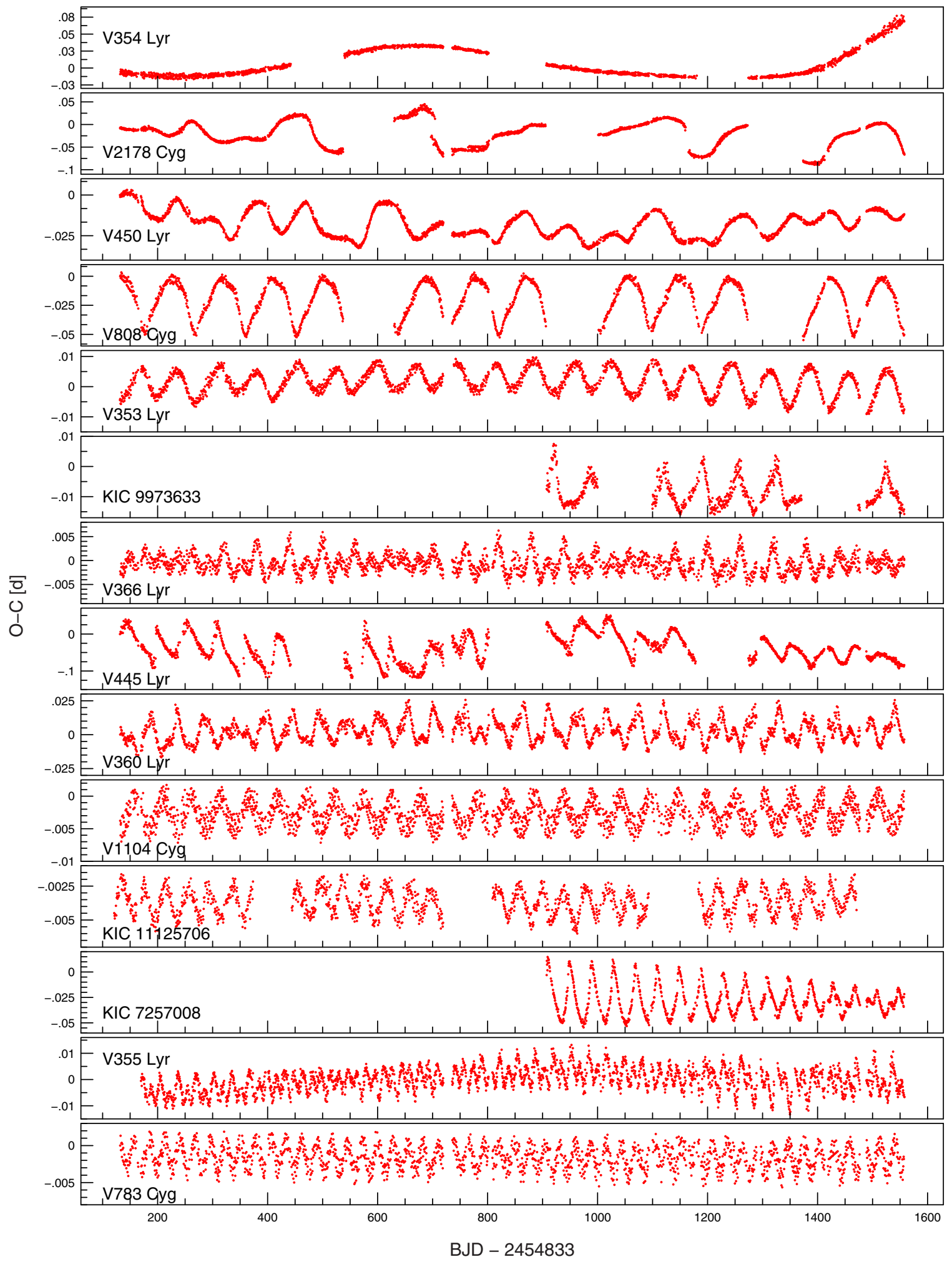

Figure 7. $O-C$ diagrams of the analyzed Blazhko RR Lyrae stars. The diagrams are constructed from interpolated times of pulsation maxima. The basic epochs are the times of the first maximum for all stars. The primary AM Blazhko period decreases from top to bottom. The LC data of V838 Cyg are not suitable for constructing the $O-C$ diagram (for details, see Section 3.2.13) and thus have not been shown here.

(A color version of this figure is available in the online journal.)

and 0.0015 day $^{-1}$ for Q10-Q16 data (for KIC 7257008 and KIC 9973633). The frequencies never change due to a modulation (Benkô et al. 2011), as opposed to the amplitudes that are affected by the FM. Consequently, our formal error estimations for the main pulsation amplitudes $(0.3-1 \mathrm{mmag})$ are lower limits only.
The Blazhko periods were determined by three different ways (see Table 2): (1) from the averaged frequency differences of the first two triplets (second column); (2) from the Blazhko frequencies themselves (Column 3 ) if they are detectable in the spectrum of the light curve; and (3) from the Fourier spectrum of the $O-C$ diagrams (Column 5). The latter two methods provide 


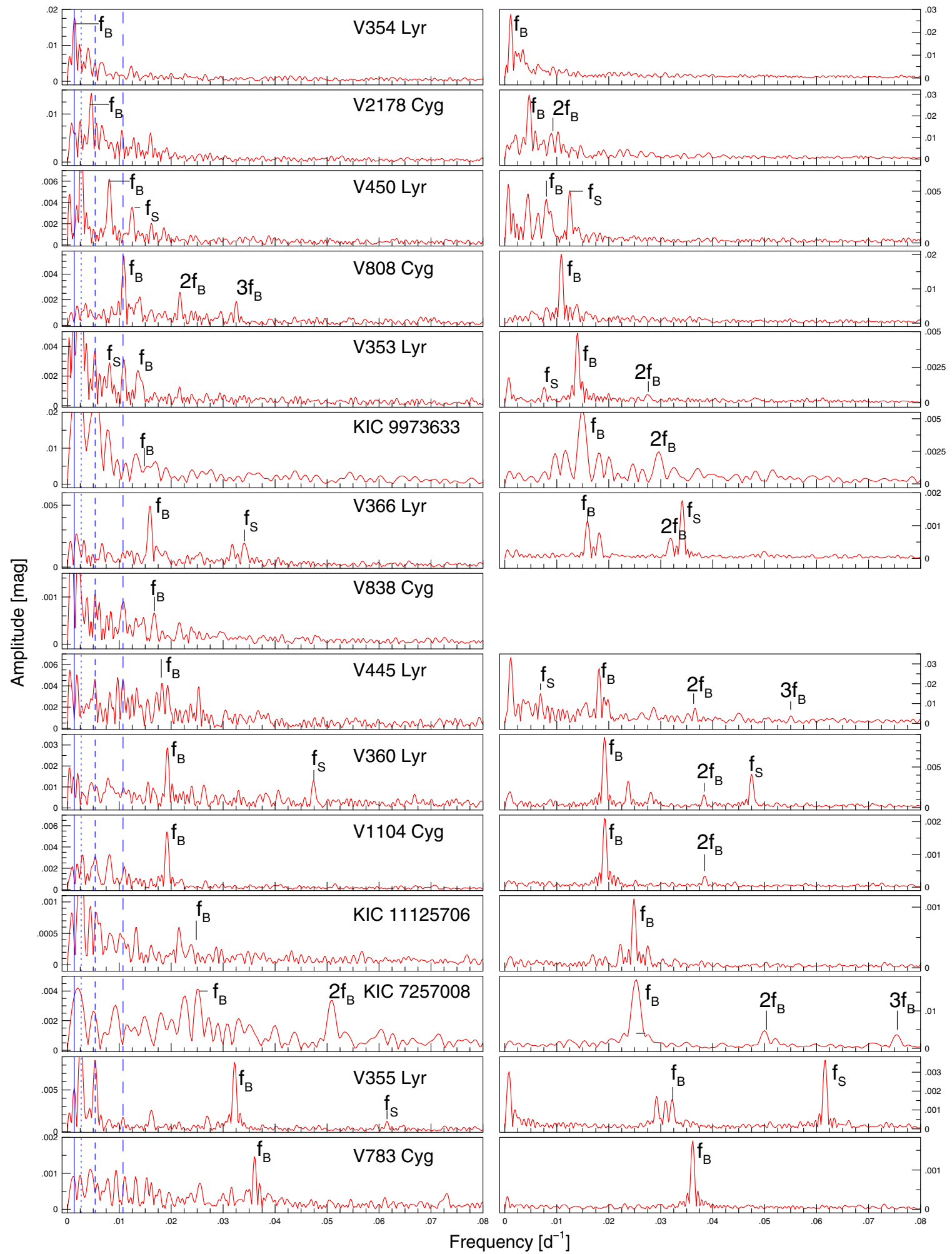

Figure 8. Properties of amplitude and frequency modulations. Left panels: low frequency range of the Fourier spectra of the light curve (Figure 4). Blue vertical lines show the location of the common instrumental frequencies: $f_{\mathrm{K}} / 2$ (continuous), $f_{\mathrm{K}}$ (dotted), $2 f_{\mathrm{K}}$ (short dashed), and $4 f_{\mathrm{K}}=f_{\mathrm{Q}}$ (long dashed), respectively. Right panels: Fourier spectra of the $O-C$ diagrams (Figure 7). For both cases the primary $\left(f_{\mathrm{B}}\right)$ and secondary modulation frequencies $\left(f_{\mathrm{S}}\right)$ and their harmonics are marked. Possible linear combinations are shown in Figures 14 and 15.

(A color version of this figure is available in the online journal.)

the AM and FM amplitudes that are shown in Columns 4 and 6 in Table 2.

We call the attention of the reader to an interesting phenomenon. In Figure 9, we plot the Blazhko period $\left(P_{i}\right)$ versus amplitude of the AM frequency $\left(A\left(f_{i}^{\mathrm{A} M}\right)\right)$ diagram, where $i=\mathrm{B}$ or $\mathrm{S}$. We find a trend in which the longer Blazhko periods mean larger amplitudes and vice versa. We cannot rule out that this is a small sample effect; however, some arguments contradict this 


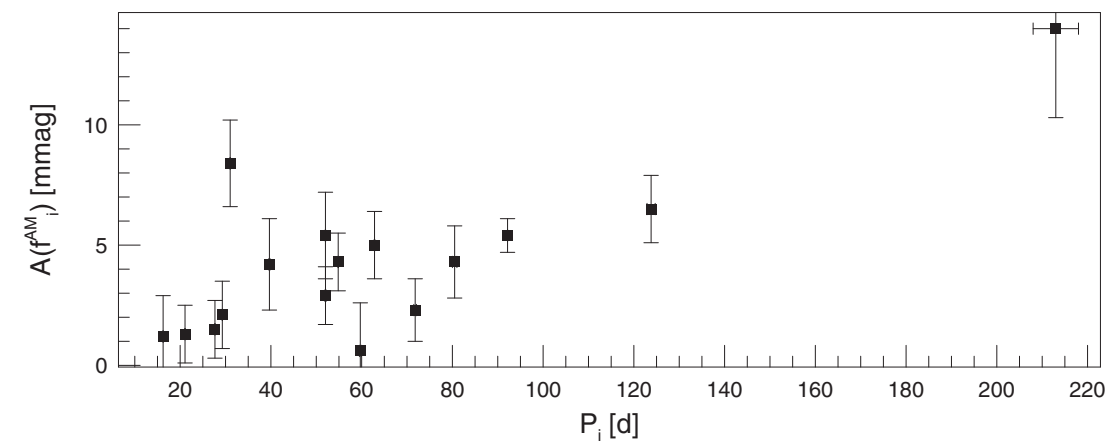

Figure 9. Blazhko period(s) vs. amplitude of the AM frequency. For the plotted values see Table 2.

Table 2

Blazhko Periods and Amplitudes from Different Methods

\begin{tabular}{|c|c|c|c|c|c|}
\hline Name & $\begin{array}{c}P_{i}^{(\mathrm{s})} \\
\text { (days) }\end{array}$ & $\begin{array}{c}P_{i}^{\mathrm{AM}} \\
\text { (days) }\end{array}$ & $\begin{array}{l}A\left(f_{i}^{\mathrm{AM}}\right) \\
(\mathrm{mmag})\end{array}$ & $\begin{array}{c}P_{i}^{\mathrm{FM}} \\
\text { (days) }\end{array}$ & $\begin{array}{c}A\left(f_{i}^{\mathrm{FM}}\right) \\
\text { (minutes) }\end{array}$ \\
\hline \multirow[t]{2}{*}{ V2178 Cyg } & $207 \pm 15$ & $216 \pm 2$ & $14 \pm 3.7$ & $215.9 \pm 0.35$ & $43.6 \pm 0.9$ \\
\hline & $168.8 \pm 1.1$ & & & $166.2 \pm 2.4$ & $18.6 \pm 1.2$ \\
\hline V808 Cyg & $92.14 \pm 0.06$ & $92.18 \pm 0.39$ & $5.4 \pm 0.7$ & $92.16 \pm 0.01$ & $30.6 \pm 0.1$ \\
\hline V783 Cyg & $27.666 \pm 0.001$ & $27.73 \pm 0.39$ & $1.5 \pm 1.2$ & $27.667 \pm 0.005$ & $2.6 \pm 0.05$ \\
\hline V354 Lyr & $807 \pm 16$ & & & $891 \pm 4$ & $36.9 \pm 0.5$ \\
\hline \multirow[t]{2}{*}{ V445 Lyr } & $54.7 \pm 0.5$ & $54.80 \pm 0.3$ & $4.3 \pm 1.2$ & $55.04 \pm 0.04$ & $38.7 \pm 1.5$ \\
\hline & $146.4 \pm 0.8$ & & & $147.4 \pm 0.7$ & $21.8 \pm 1.7$ \\
\hline KIC 7257008 & $39.51 \pm 0.05$ & $39.7 \pm 0.4$ & $4.2 \pm 1.9$ & $39.72 \pm 0.02$ & $26.3 \pm 0.4$ \\
\hline \multirow[t]{2}{*}{ V355 Lyr } & $31.06 \pm 0.1$ & $31.04 \pm 0.08$ & $8.4 \pm 1.8$ & $30.99 \pm 0.02$ & $2.3 \pm 0.1$ \\
\hline & $16.243 \pm 0.007$ & $16.25 \pm 0.1$ & $1.2 \pm 1.7$ & $16.229 \pm 0.003$ & $5.2 \pm 0.1$ \\
\hline \multirow[t]{2}{*}{ V450 Lyr } & $123.7 \pm 0.4$ & $123.0 \pm 1$ & $6.5 \pm 1.4$ & $124.8 \pm 0.3$ & $5.7 \pm 0.3$ \\
\hline & $81.0 \pm 0.6$ & $80.4 \pm 0.8$ & $4.3 \pm 1.5$ & $80.1 \pm 0.1$ & $6.7 \pm 0.3$ \\
\hline \multirow[t]{2}{*}{ V353 Lyr } & $71.70 \pm 0.04$ & $72.1 \pm 1.5$ & $2.3 \pm 1.3$ & $71.68 \pm 0.02$ & $7.17 \pm 0.07$ \\
\hline & $133.1 \pm 0.4$ & & & $131.3 \pm 0.3$ & $1.64 \pm 0.08$ \\
\hline \multirow[t]{2}{*}{ V366 Lyr } & $62.90 \pm 0.01$ & $62.87 \pm 0.4$ & $5.0 \pm 1.4$ & $62.77 \pm 0.05$ & $1.66 \pm 0.06$ \\
\hline & $29.29 \pm 0.01$ & $29.28 \pm 0.3$ & $2.1 \pm 1.4$ & $29.295 \pm 0.007$ & $2.58 \pm 0.06$ \\
\hline \multirow[t]{2}{*}{ V360 Lyr } & $52.10 \pm 0.01$ & $51.88 \pm 0.5$ & $2.9 \pm 1.2$ & $52.11 \pm 0.015$ & $12.9 \pm 0.2$ \\
\hline & $21.041 \pm 0.008$ & $21.09 \pm 0.15$ & $1.3 \pm 1.2$ & $21.073 \pm 0.005$ & $6.0 \pm 0.2$ \\
\hline \multirow[t]{2}{*}{ KIC 9973633} & $67.11 \pm 0.08$ & & & $67.30 \pm 0.07$ & $8.2 \pm 0.2$ \\
\hline & $27.13 \pm 0.06$ & & & $27.21 \pm 0.15$ & $1.6 \pm 0.4$ \\
\hline V838 Cyg & $59.5 \pm 0.1$ & $59.8 \pm 3$ & $0.6 \pm 2$ & & \\
\hline \multirow[t]{2}{*}{ KIC 11125706} & $40.21 \pm 0.02$ & & & $40.21 \pm 0.01$ & $1.66 \pm 0.03$ \\
\hline & & & & $58.9 \pm 0.1$ & $0.27 \pm 0.03$ \\
\hline V1104 Cyg & $52.00 \pm 0.01$ & $52.08 \pm 0.2$ & $5.4 \pm 1.8$ & $51.99 \pm 0.02$ & $3.14 \pm 0.05$ \\
\hline
\end{tabular}

Notes. $P_{i}$ and $A\left(f_{i}\right)$ denote the Blazhko periods and the amplitude of the modulation frequencies, where $i=\mathrm{B}$ or $\mathrm{S}$ for primary and secondary Blazhko periods, respectively. The upper indices denote the method of the calculation: (s), from the side peaks around harmonics; AM, direct detection in the light curve spectra; FM, from the spectra of the $O-C$ diagrams.

scenario. The emptiness of the long-period and small-amplitude part of the diagram can be explained by the observational effect (it is difficult to distinguish between small-amplitude, longperiod stellar variations and the instrumental effects with similar timescales, even in the Kepler data), but the lack of points of the small-period and large-amplitude part cannot. Additionally, similar effects are common for (hydro)dynamical systems: e.g., weakly dissipating systems could be perturbed for high amplitude by long-timescale perturbing forces only (Molnár et al. 2012b). The found effect will be investigated in a separate study.

Basic parameters obtained from this analysis are summarized in Table 3. The columns of the table show the ID numbers and names of the stars, main pulsation periods, and their Fourier amplitude, where the number of digits indicate the accuracy. Columns 5 and 6 contain the modulation periods averaged from the values in Table 2 . The last two columns of the table indicate the presence of additional frequencies, instrumental problems, and auxiliary information about the Kepler observations.

\subsection{Analysis of Individual Stars$$
\text { 3.2.1. V2178 Cyg }=\text { KIC } 3864443
$$

This star was chosen by $\mathrm{N} 13$ as being representative of the long-period Blazhko stars showing large AM and FM. The envelope of the light curve in Figure 4 shows complicated amplitude changes suggesting multiperiodicity and/or cycle-tocycle variations of the Blazhko effect. Unfortunately, the long timescales of the variations make quantification of this phenomenon impossible. We detected 11 significant harmonics of the main pulsation frequency $\left(f_{0}\right)$ up to the Nyquist frequency. The triplet structures around the harmonics are highly asymmetric: $A\left(k f_{0}-f_{\mathrm{B}}\right) \gg A\left(k f_{0}+f_{\mathrm{B}}\right)$ (see also Figure 3 in N13). If we calculate the primary Blazhko frequency from the averaged spacing of the side peaks, we find $0.00482 \mathrm{day}^{-1}$. This value is in good agreement with the highest amplitude peak in the low frequency range $\left(0.00462 \pm 0.0001\right.$ day $\left.^{-1}\right)$, so it can be identified with $f_{\mathrm{B}}$ (Figure 8 ). Due to the missing quarters, 

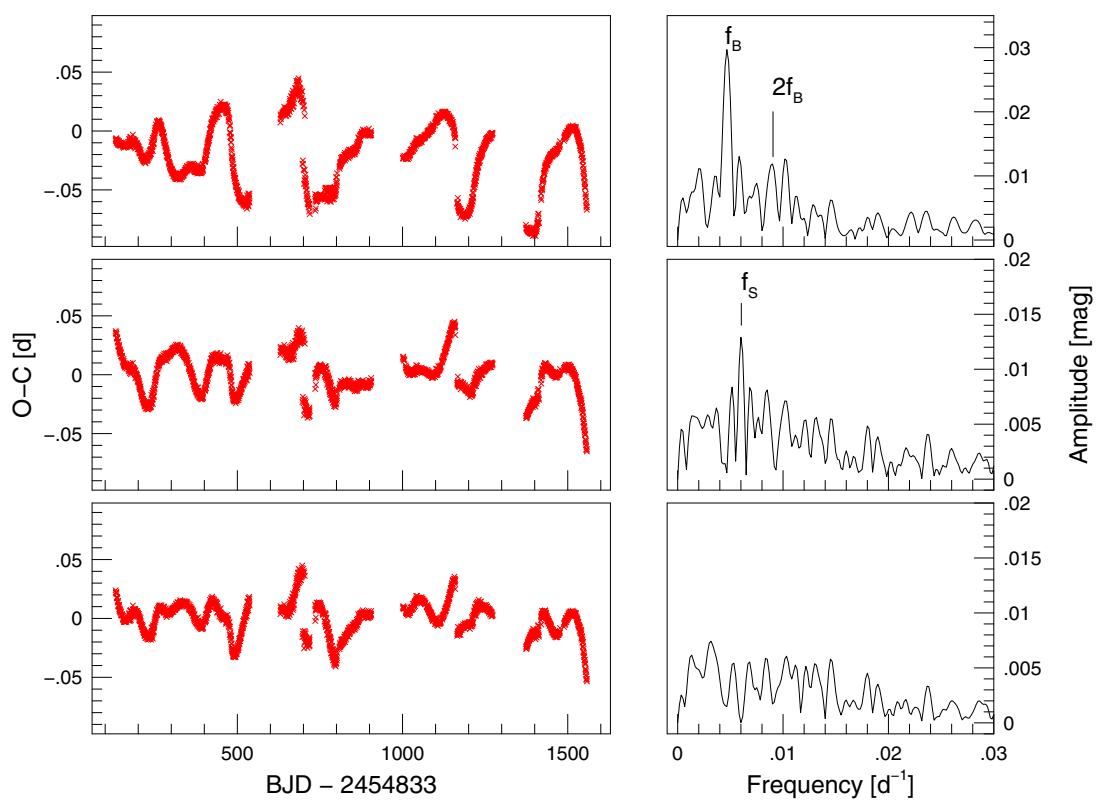

Figure 10. $O-C$ analysis of $\mathrm{V} 2178 \mathrm{Cyg}$. Left panels show the $O-C$ diagram (top) and its residuals after we pre-whitened the data with $f_{\mathrm{B}}$ and $2 f_{\mathrm{B}}(\mathrm{middle})$ and also with $f_{\mathrm{S}}$ (bottom). Right panels show the Fourier spectra of the $O-C$ data during this consecutive pre-whitening process.

(A color version of this figure is available in the online journal.)

Table 3

Basic Properties of the Kepler Blazhko Stars

\begin{tabular}{|c|c|c|c|c|c|c|c|}
\hline KIC & GCVS & $\begin{array}{c}P_{0} \\
\text { (days) }\end{array}$ & $\begin{array}{l}A\left(f_{0}\right) \\
(\mathrm{mag})\end{array}$ & $\begin{array}{c}P_{\mathrm{B}} \\
\text { (days) }\end{array}$ & $\begin{array}{c}P_{\mathrm{S}} \\
\text { (days) }\end{array}$ & Add. Freq. ${ }^{a}$ & Remarks $^{\mathrm{b}}$ \\
\hline 3864443 & V2178 Cyg & 0.4869470 & 0.3156 & $213 \pm 5$ & $167.5 \pm 1.8(?)$ & $\mathrm{F} 2,(\mathrm{PD})$ & $\mathrm{m}$ \\
\hline 4484128 & V808 Cyg & 0.5478635 & 0.2197 & $92.16 \pm 0.02$ & $\sim 1000$ & $\mathrm{PD},(\mathrm{F} 2)$ & $\mathrm{m}$ \\
\hline 5559631 & V783 Cyg & 0.6207001 & 0.2630 & $27.6667 \pm 0.0005$ & & & scal \\
\hline 6183128 & V354 Lyr & 0.5616892 & 0.2992 & $849 \pm 59$ & (?) & $\mathrm{F} 2,\left(\mathrm{PD}, \mathrm{F}^{\prime}\right)$ & scal, m \\
\hline 6186029 & V445 Lyr & 0.5130907 & 0.2102 & $54.83 \pm 0.04$ & $146.9 \pm 0.7$ & $\mathrm{PD}, \mathrm{F} 1, \mathrm{~F} 2$ & $\mathrm{~m}$ \\
\hline 7257008 & & 0.511787 & 0.2746 & $39.67 \pm 0.14$ & $>900$ & $\mathrm{PD}, \mathrm{F} 2$ & Q10- \\
\hline 7505345 & V355 Lyr & 0.4736995 & 0.3712 & $31.02 \pm 0.05$ & $16.24 \pm 0.01$ & $\mathrm{PD}, \mathrm{F} 2$ & \\
\hline 7671081 & V450 Lyr & 0.5046198 & 0.3110 & $123.8 \pm 0.9$ & $80.5 \pm 0.5$ & F2 & scal \\
\hline 9001926 & V353 Lyr & 0.5567997 & 0.2842 & $71.8 \pm 0.3$ & $132.2 \pm 1.3$ & & \\
\hline 9578833 & V366 Lyr & 0.5270284 & 0.2909 & $62.84 \pm 0.07$ & $29.29 \pm 0.01$ & (F2) & \\
\hline 9697825 & V360 Lyr & 0.5575755 & 0.2572 & $52.03 \pm 0.14$ & $21.07 \pm 0.03$ & $\mathrm{~F} 2,(\mathrm{PD})$ & \\
\hline 9973633 & & 0.510783 & 0.2458 & $67.2 \pm 0.1$ & $27.17 \pm 0.06$ & $\mathrm{PD}, \mathrm{F} 2$ & m, Q10- \\
\hline 10789273 & V838 Cyg & 0.4802800 & 0.3909 & $59.7 \pm 0.2$ & & $(\mathrm{~F} 2, \mathrm{PD})$ & scal \\
\hline 11125706 & & 0.6132200 & 0.1806 & $40.21 \pm 0.02$ & $58.9 \pm 0.1$ & & $\mathrm{~m}$, scal \\
\hline 12155928 & V1104 Cyg & 0.4363851 & 0.3847 & $52.02 \pm 0.05$ & & & scal \\
\hline
\end{tabular}

Notes. $P_{0}, P_{\mathrm{B}}$, and $P_{\mathrm{S}}$ mean the pulsation and the primary and secondary modulation periods, respectively. $A\left(f_{0}\right)$ is the Fourier amplitude of the main pulsation frequency.

${ }^{a}$ The pattern of additional frequencies: PD means period doubling; F1 indicates first overtone frequency and its linear combination with the fundamental one; F2 is as F1, but with the second radial overtone; $\mathrm{F}^{\prime}$ indicates frequencies with unidentified modes; and brackets indicate marginal effects.

${ }^{\mathrm{b}} \mathrm{scal}=$ scaled, $\mathrm{m}=$ missing quarters, $\mathrm{Q} 10-=$ data from $\mathrm{Q} 10$.

the Fourier spectrum contains numerous instrumental frequencies such as $f_{\mathrm{Q}}$ and $f_{\mathrm{Q}} \pm f_{\mathrm{B}}$ and their linear combinations with the main frequency and its harmonics. The second largest low frequency peak is at $0.002486 \mathrm{day}^{-1}$, which coincides with $f_{\mathrm{K}}=0.002685 \mathrm{day}^{-1}$ within the determination error $\left(0.0001\right.$ day $\left.^{-1}\right)$.

After we subtracted the largest amplitude side peaks $\left(k f_{0}-\right.$ $\left.f_{\mathrm{B}}\right)$ in the spectrum of the residual, the other components of the triplets $\left(k f_{0}+f_{\mathrm{B}}\right)$ and additional side peaks of a possible secondary modulation $\left(k f_{0}-f_{\mathrm{S}}\right)$ appeared, where $f_{\mathrm{S}}=0.00593 \mathrm{day}^{-1}$. If $f_{\mathrm{S}}$ belongs to a secondary modulation, the ratio of two modulation frequencies would be 2:3; however, $f_{\mathrm{S}}$ can also be interpreted as a linear combination: $f_{\mathrm{S}}=f_{\mathrm{Q}}-f_{\mathrm{B}}$.

When we compute the Fourier spectrum of the $O-C$ diagram (Figure 10), we find $f_{\mathrm{B}}=0.00463 \mathrm{day}^{-1}$ and $2 f_{\mathrm{B}}$. When we pre-whiten with these frequencies, one significant peak appears at $0.00602 \mathrm{day}^{-1}(\mathrm{~S} / \mathrm{N}=24)$. If we remove this frequency as well, the residual curve shows large amplitude quasi-periodic oscillations, but no further frequencies can be identified. Our conclusion is that V2178 Cyg shows a multiperiodic and/or quasi-periodic Blazhko effect, but the long periods do not allow us to draw a final conclusion. 
Similar to Benkő et al. (2010), we found a bunch of peaks around the frequency of the second radial overtone mode (see also in Figure $6, f_{2}=3.51478$ day $^{-1}, P_{0} / P_{2}=0.584$; $\mathrm{S} / \mathrm{N} \approx 3)$. The PD phenomenon is marginal: the highest peak around $1.5 f_{0}$ is $f^{(1)}=3.05804 \mathrm{day}^{-1}\left(f^{(1)} / f_{0}=1.49\right.$; $\mathrm{S} / \mathrm{N} \approx 2$ ). A third additional peak condensation can be seen around $f^{(2)}=2.656875 \mathrm{day}^{-1}(\mathrm{~S} / \mathrm{N} \approx 2)$. Though some Blazhko stars (e.g., RR Lyr, V445 Lyr) show a radial first overtone frequency $\left(f_{1}\right)$ around this region, we could identify this peak of V2178 Cyg as a linear combination of $f^{(2)}=3 f_{0}-f_{2}$ with high certainty, because the period ratio of $P^{(2)} / P_{0}=0.773$ is far from the canonical value of $P_{1} / P_{0}=0.744$. Because this period ratio increases with the increasing metallicity (see, e.g., Figure 8 in Chadid et al. 2010), the measured low metallicity of V2178 Cyg $([\mathrm{Fe} / \mathrm{H}]=-1.46 ; \mathrm{N} 13)$ also supports the linear combination explanation.

\subsection{2. $V 808$ Cyg $=$ KIC 4484128}

The light curve of this star in Figure 4 shows two important features. First, the envelope shape suggests a highly nonsinusoidal AM. Second, the length of the Blazhko cycle is close to the length of the observing quarters. As a consequence of the first fact, we can detect two significant harmonics, $2 f_{\mathrm{B}}$ and $3 f_{\mathrm{B}}$, of the Blazhko frequency $f_{\mathrm{B}}=0.01085 \mathrm{day}^{-1}$ (Figure 8), and multiplet side peaks $\left(k f_{0} \pm l f_{\mathrm{B}}\right.$, where $\left.l>1\right)$ are detectable as well. A slight cycle-to-cycle amplitude change might be present, but the quarter-long Blazhko period and gaps together make such an effect barely detectable.

The $O-C$ diagram of V808 Cyg can be fitted well with the Blazhko period and its three harmonics. After we subtracted this four-frequency fit from the $O-C$ data, a definite structure can be detected in the residual spectrum (panel (B) in Figure 11). At the positions of $f_{\mathrm{B}}$ and $3 f_{\mathrm{B}}$, side peaks appear. These peaks define a secondary modulation with the frequency of $f_{\mathrm{S}}=0.0010 \mathrm{day}^{-1}$. The pre-whitened spectrum indeed shows two peaks at $f_{\mathrm{S}}$ and $2 f_{\mathrm{S}}$ (panel (C)). However, the possible period $P_{\mathrm{S}} \sim 1000$ days is commensurable with the length of the total observational time, so this $O-C$ variation in panel (D) could be secular as well.

V808 Cyg shows the strongest known PD effect, which is why data taken during the first two quarters were investigated in detail by Szabó et al. (2010). Using the time series up to Q16, this main finding remains unchanged. The highest amplitude HIF is at $1.5 f_{0}$, namely, $f^{(1)}=2.69770$ day $^{-1}\left(f^{(1)} / f_{0}=1.48\right.$; $\mathrm{S} / \mathrm{N} \approx 30$ ). After applying a pre-whitening process that is completed in a few steps, i.e., we subtract the main pulsation frequency, its harmonics, and some (6-10) significant multiplets around the harmonics, we found that the second radial overtone mode (or a non-radial mode at the location of the radial one) $f_{2}=3.09774$ day $^{-1}\left(P_{2} / P_{0}=0.589\right)$ is also excited (panel (E) in Figure 11). The amplitude of this frequency is much lower than the amplitudes of the PD frequencies, which explains why previous investigations have not discovered this mode.

$$
\text { 3.2.3. } V 783 \text { Cyg }=\text { KIC } 5559631
$$

The Blazhko effect of V783 Cyg seems to be simple: a sinusoidal AM and FM visible both in the light curve (Figure 4) and $O-C$ diagram (Figure 7). By investigating these curves more carefully, we can detect small differences between consecutive cycles.

When we pre-whiten the light curve data with the main pulsation frequency and its 15 significant harmonics, we find nice symmetric triplet structures in the spectrum around the
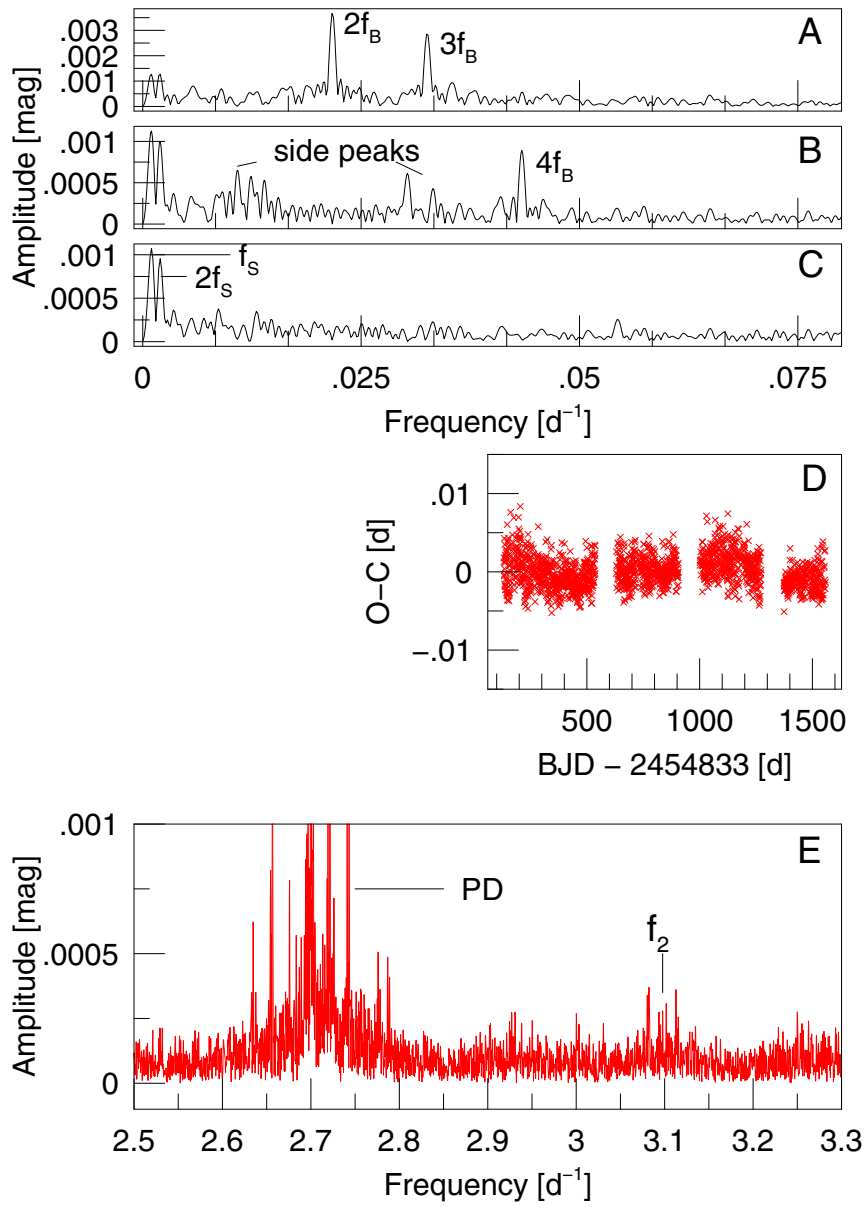

Figure 11. New results from V808 Cyg. Panels (A)-(C): the pre-whitening process of the $O-C$ diagram shown in Figure 7. The harmonics of the Blazhko frequency $f_{\mathrm{B}}$ are significant up to the fourth order. A long-timescale variation is evident because of the presence of low frequency peaks $\left(f_{\mathrm{S}}, 2 f_{\mathrm{S}}\right)$ and side frequencies around the harmonics of $f_{\mathrm{B}}$. Panel (D): residual $O-C$ curve containing only $f_{\mathrm{S}}$ and $2 f_{\mathrm{S}}$. Panel $(\mathrm{E})$ : the second radial overtone frequency $\left(f_{2}\right)$ can be detected from the Q1-Q16 data.

(A color version of this figure is available in the online journal.)

harmonics. The star has the shortest Blazhko cycle in our sample $\left(P_{\mathrm{B}}=27.67\right.$ days). The spectrum also contains the modulation frequency itself: $f_{\mathrm{B}}=0.036058 \mathrm{day}^{-1}$. After subtracting the triplets in the residual spectrum, multiplet side frequencies appear. By carrying out a similar pre-whitening process as in Section 3.2.2, we can eliminate all side peaks and no additional peaks emerge between the harmonics.

Fourier analysis of the $O-C$ diagram provides us $f_{\mathrm{B}}$ again (Figure 8). When we subtract a fit with $f_{\mathrm{B}}$, the residual $O-C$ diagram shows a parabolic shape indicating a period change. Fitting a quadratic function in the form

$$
O-C=\frac{1}{2} \frac{d P_{0}}{d t} \bar{P}_{0} E^{2}
$$

(Sterken 2005), where $\bar{P}_{0}$ means the averaged period and $E$ the cycle number from the initial epoch, we find a period increase: $d P_{0} / d t=1.02 \times 10^{-9} \pm 1.7 \times 10^{-10}$ dday $^{-1}$. That is, $0.12 \pm 0.02$ $\mathrm{dMy}^{-1}$, which agrees well with the value of Cross (1991), $0.088 \pm 0.023 \mathrm{dMy}^{-1}$, which was determined on the basis of photometric observations between 1933 and 1990.

The Fourier analysis of the light curve and $O-C$ diagram is not sensitive to the mentioned slight cycle-to-cycle variation of the Blazhko effect. The short Blazhko period and uninterrupted 


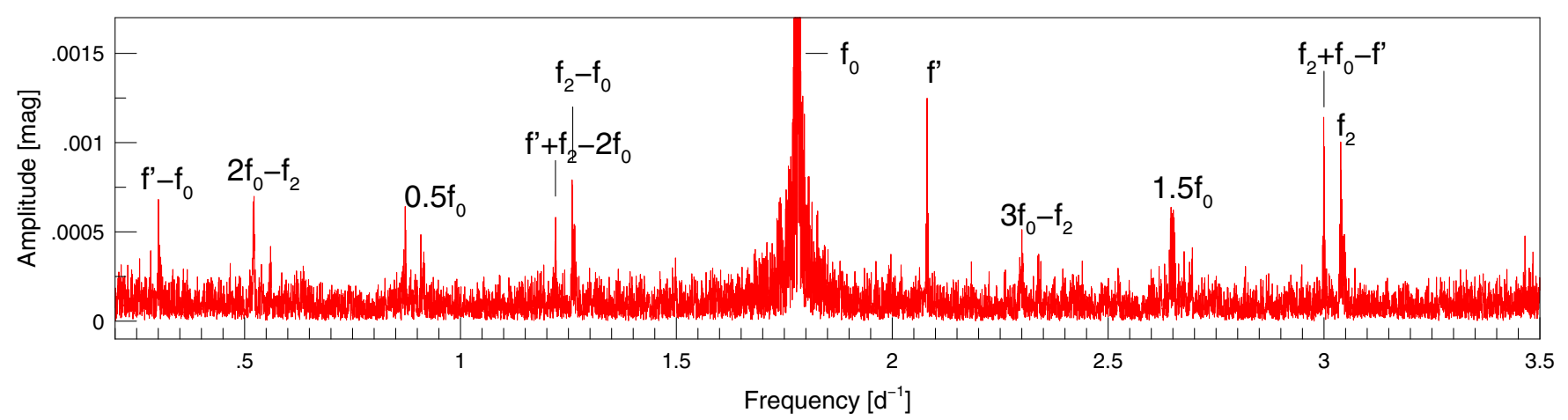

Figure 12. Additional peaks in the lower frequency range of the pre-whitened Fourier spectrum of the V354 Lyr light curve.

(A color version of this figure is available in the online journal.)

Kepler data make V783 Cyg a good candidate for a dynamical analysis. This study will be discussed in a separate paper by E. Plachy et al. (in preparation). The preliminary results suggest that the cycle-to-cycle variation of the V783 Cyg light curve and $O-C$ diagram have a chaotic nature.

$$
\text { 3.2.4. V354 Lyr }=\text { KIC } 6183128
$$

This star has the longest Blazhko period in the Kepler sample. We find $P_{\mathrm{B}}=807$ days $\left(f_{\mathrm{B}}=0.00124\right.$ day $\left.^{-1}\right)$, which we calculated from the triplet spacing in the spectrum of the light curve. At the same time, the highest peak in the low frequency range is at $f_{\mathrm{B}}=0.00134$ day $^{-1}$, which yields $P_{\mathrm{B}}=748$ days. The problem is that the Blazhko period is inseparably close to the instrumental period $2 P_{\mathrm{K}}=745 \mathrm{~d}$.

The two observed Blazhko cycles in Figure 4 look different. The ascending branch of the first cycle is steeper than that of the second one, while the descending branch of the second cycle is steeper. The different shapes of the two cycles in the $O-C$ diagram (Figure 7) strengthen our suspicion: V354 Lyr shows multiperiodicity or cycle-to-cycle variations in the Blazhko effect. The long Blazhko period prevents us from quantifying this feature.

As it was already found by Benkô et al. (2010), the Fourier spectrum of V354 Lyr contains significant additional peaks between harmonics. The following frequencies were reported between $f_{0}$ and $2 f_{0}$ (with the notations of the referred paper): $f^{\prime}=2.0810, f^{\prime \prime}=2.4407, f^{\prime \prime \prime}=2.6513$, and $f_{2}=$ $3.03935 \mathrm{day}^{-1}$. After we removed the main pulsation frequency, its significant harmonics, and the highest amplitude side peaks around the harmonics, our spectrum (Figure 12) also contains numerous additional frequencies. The highest amplitude peak from the HIF is located now at $f^{(1)}=2.648387=1.5 f_{0}$ $\left(f^{(1)} / f_{0}=1.49\right)$. Identifying the frequency of the radial second overtone mode is problematic, because there is a double peak in that position: $f_{2}^{(1)}=3.038671 \mathrm{day}^{-1}\left(P_{2}^{(1)} / P_{0}=0.586\right)$ and $f_{2}^{(2)}=2.999333$ day $^{-1}\left(P_{2}^{(1)} / P_{0}=0.593\right)$. The spacing between these two frequencies is $0.300 \mathrm{day}^{-1}$. Similar double peak structures can be seen for many other frequencies.

The highest amplitude additional frequency $f^{\prime}=$ 2.080672 day $^{-1}$ is mysterious. We have not found any frequencies in such a position for other Blazhko stars (see also Figure 6). Neither known instrumental frequencies nor linear combinations of instrumental and real frequencies give a peak at this position. The spectral window function has a comb-like structure (Van Cleve et al. 2009), but the peaks are far from $f^{\prime}$ and their amplitude are very low (in a normalized scale $\sim 0.004$ ). So we can rule out $f^{\prime}$ being an instrumental frequency.
We checked whether this frequency comes from this star or not. Using the flux data, we searched for its linear combination frequencies with the main pulsation one. Both the $f^{\prime}+f_{0}=$ $3.8613 \mathrm{day}^{-1}$ and $f^{\prime}-f_{0}=0.3003 \mathrm{day}^{-1}$ are indeed detectable. So we could rule out a background star as the source of this frequency. The period ratio is $P^{\prime} / P_{0}=0.855$. Benkő \& Szabó (2014) provided an explanation that this frequency would be a linear combination of the main pulsation $f_{0}$ and radial first overtone mode frequencies $f_{1}$, namely, $f^{\prime}=\left(f_{0}+f_{1}\right) / 2$. The problem of this interpretation is that the spectrum shows significant peaks neither at $f_{0}+f_{1}$ nor at $f_{1}$.

As we have seen, the spacing between $f_{2}^{(1)}$ and $f_{2}^{(2)}$ can be given as $f^{\prime}-f_{0}$, so any of them can be calculated as a linear combination of $f_{2}, f_{0}$ and $f^{\prime}$. In Figure 12, we identified $f_{2}=f_{2}^{(1)}$; therefore, $f_{2}^{(2)}=f_{2}+f_{0}-f^{\prime}$. Alternatively, if we identify $f_{2}=f_{2}^{(2)}$, then $f_{2}^{(1)}=f_{2}+f^{\prime}-f_{0}$. We can find several other peaks where we have alternate identifications depending on how we choose $f_{2}$.

The last frequency listed by Benkô et al. (2010) is $f^{\prime \prime}=$ $2.4407 \mathrm{day}^{-1}$. Although this frequency is not significant in the spectrum calculated from Q1-Q16 data, half of it $\left(1.220\right.$ day $\left.^{-1}\right)$ can be detected. The later frequency can easily be produced by $f_{2}-f_{0}$ if we identify $f_{2}=f_{2}^{(2)}$. As we have shown in Benkő \& Szabó (2014), the frequency combination $2\left(f_{2}-f_{0}\right)$ could explain numerous previously unidentified peaks in spectra of some CoRoT and Kepler Blazhko stars. So it is not surprising that we temporarily detect this combination frequency $2\left(f_{2}-\right.$ $\left.f_{0}\right)=2.4407 \mathrm{day}^{-1}$ in V354 Lyr as well. As we mentioned in Section 3.1.1, all additional frequency amplitudes (HIFs, overtones) change in time, and these combination frequencies also seem to show a similar time dependency.

\subsection{5. $V 445 \mathrm{Lyr}=\mathrm{KIC} 6186029$}

The light curve of this star shows strong and complicated amplitude changes (Figure 4). It was the subject of a detailed study by Guggenberger et al. (2012). The paper used the data available in that time (Q1-Q7), but its main statements are not changed in light of the more extended Q1-Q16 data set. The heavily varying parameters such as the different periods, amplitudes, and phases result in slightly different averaged values for these parameters compared to ones given by Guggenberger et al. (2012). We confirm the existence of two modulation frequencies $\left(f_{\mathrm{B}}, f_{\mathrm{S}}\right)$ and four additional frequency patterns, namely, $f_{2}, f_{1}, \mathrm{PD}$, and $f_{\mathrm{N}}=2.763622$ day $^{-1}$. In the latter case, we noted a possible interpretation with the linear combination of $f_{\mathrm{N}}=2\left(f_{2}-f_{0}\right)$ in Benkő \& Szabó (2014). 


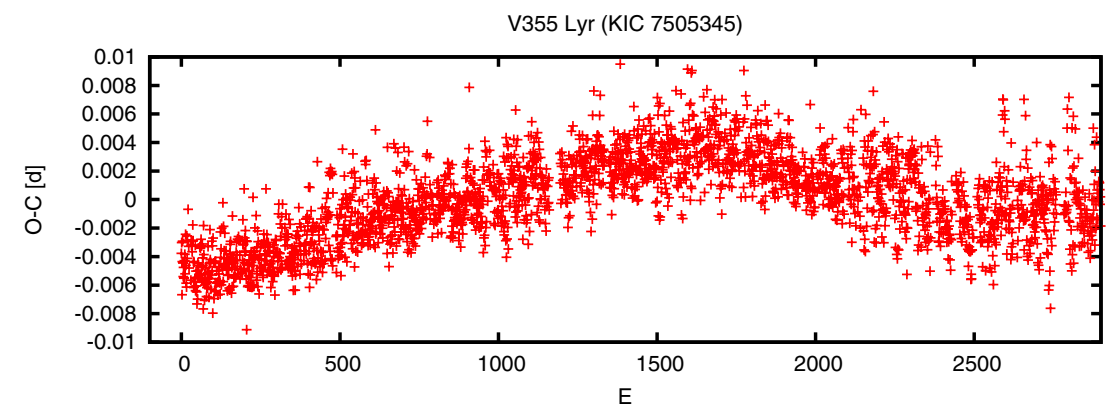

Figure 13. Residual $O-C$ diagram of $\mathrm{V} 355 \mathrm{Lyr}$ after we pre-whitened the data with the frequencies $k f_{\mathrm{B}}$ and $(k=1,2,3,5)$ and the side peaks $f_{\mathrm{B}} \pm f_{\mathrm{L}}$. (A color version of this figure is available in the online journal.)

\subsubsection{KIC 7257008}

The variable nature of this star was discovered by the ASAS survey (Pojmanski 1997, 2002; R. Szabó et al., in preparation). Its Kepler data were investigated for the first time by N13. The envelope of the light curve in Figure 4 suggests multiple modulation behavior. We determined the Blazhko frequency both from the side peak patterns and from the low frequency range of the Fourier spectrum of the light curve: $f_{\mathrm{B}}$ $=0.02528 \mathrm{day}^{-1}$. The harmonic $2 f_{\mathrm{B}}$ is also significant as a consequence of the non-sinusoidal nature of the AM.

The FM is even more non-sinusoidal: the Fourier spectrum of the $O-C$ diagram (in Figure 8) contains five significant harmonics of the Blazhko frequency. A small peak at the subharmonic $f_{\mathrm{B}} / 2=0.01234 \mathrm{day}^{-1}(\mathrm{~S} / \mathrm{N}=3.6)$ is also present. After pre-whitening the Fourier spectra of the upper envelope (maxima) curve or $O-C$ diagram, double side peaks remain at the location of the Blazhko frequency and its harmonics. The spacing between side peaks is very narrow $\left(\sim 0.001 \mathrm{day}^{-1}\right)$, which implies a secondary Blazhko period longer than the length of the data set (Q10-Q16). The amplitude and the phase of the harmonics of $f_{\mathrm{B}}$ have changed during the observing term, producing varying envelope and $O-C$ curves. These changes have been verified by using PERIOD04 amplitude and phase variation tool. Molnár et al. (2014) have found that the star shows the PD effect $\left(1.5 f_{0}=2.871047 \mathrm{day}^{-1}\right)$ and contains the second overtone pulsation $\left(f_{2}=3.329353\right.$ day $\left.^{-1}\right)$ as well.

$$
\text { 3.2.7. V355 Lyr }=\text { KIC } 7505345
$$

The light curve of V355 Lyr in Figure 4 suggests at least two modulation periods. The longer period amplitude change shows about four cycles during the four year observing span. It raises the possibility of an instrumental effect showing the Kepler year (372.5 days; Bányai et al. 2013). Indeed, we found two strong peaks in the low frequency range of the spectrum, which can be identified as $f_{\mathrm{K}}=0.00266$ and $2 f_{\mathrm{K}}=$ $0.00533 \mathrm{day}^{-1}$. However, other aspects contradict such an explanation. The Blazhko frequency turns up from the triplet structures, and it is detectable directly as $f_{\mathrm{B}}=0.0322 \pm$ 0.005 day $^{-1}$. There is a detectable peak at $f=0.06154$ day $^{-1}$ $(\mathrm{S} / \mathrm{N}=4.5)$ which cannot be the harmonic $2 f_{\mathrm{B}}$, because its difference from the exact harmonic $\left(0.00147\right.$ day $\left.^{-1}\right)$ is twice of the Rayleigh frequency resolution $\left(\approx 0.0007 \mathrm{day}^{-1}\right)$. So we may identify it as a possible secondary modulation frequency ( $\left.f=f_{\mathrm{S}}\right)$. If this is true, the two modulation frequencies have a ratio of nearly $1: 2$, causing the observed beating phenomenon in the envelope curve.

At the same time, the sub-harmonic $f_{\mathrm{B}} / 2$ is also significant. A similar situation has been found for the first time by Sódor et al. (2011) in the case of the multiperiodic Blazhko star CZ Lac. Jurcsik et al. (2012) also detected the sub-harmonic of the Blazhko frequency for RZ Lyr. As Sódor et al. (2011) discussed, we can also identify $f_{\mathrm{B}} / 2$ as the primary modulation frequency. In that case, instead of a sub-harmonic we would have a harmonic with a much higher amplitude than the main modulation frequency. Moreover, the modulation curve would have a rather unusual shape. For these reasons we prefer the sub-harmonic identification.

As opposed to the above-mentioned amplitude relations, the Fourier amplitude $A\left(f_{\mathrm{S}}\right)$ is higher than $A\left(f_{\mathrm{B}}\right)$ in the spectrum of the $O-C$ curve. In other words, in the FM the $f_{\mathrm{S}}$ dominates, while in the AM the $f_{\mathrm{B}}$ is the dominant. Such an effect have never been detected before. Linear combination peaks at $f_{\mathrm{S}} \pm f_{\mathrm{B}}$ are also detectable. Additionally, the subharmonic of $f_{\mathrm{B}}$ cannot be seen, but the sub-harmonic of $f_{\mathrm{S}}=0.03083 \mathrm{day}^{-1}$ can be detected. One other significant peak is at $f^{(1)}=0.16316$ day $^{-1}(\mathrm{~S} / \mathrm{N}=5.3)$. This frequency is located close to $5 f_{\mathrm{B}}=0.16150 \mathrm{day}^{-1}$ but the identification $f^{(1)}=5 f_{\mathrm{B}}$ is ambiguous, because no other harmonics are detectable. The spectrum of the light curve also shows a marginal $(\mathrm{S} / \mathrm{N}=2.4)$ peak at this position. We suspect that it might be a third modulation frequency. By pre-whitening the $O-C$ curve with all the above mentioned frequencies, we obtain the residual curve in Figure 13. This residual shows a sudden period change at about $E \approx 1636(=\mathrm{BJD} \approx 2455778)$ and a less pronounced one around $E \approx 2386(=\mathrm{BJD} \approx 2456133)$. Nothing in particular can be seen in the light curves around these dates.

The higher frequency range of the time series data is dominated by the main pulsation frequency, its harmonics, and their strong multiplet surroundings. Beyond the clear PD effect $\left(1.5 f_{0}=3.155484\right.$ day $\left.^{-1} ; P / P_{0}=1.495\right)$ discussed by Szabó et al. (2010), the Fourier spectrum of the star shows evidence for the second radial overtone pulsation (see in Figure 6). This feature was undetected by previous Kepler studies. The frequency $f_{2}=3.589528$ day $^{-1}\left(P_{2} / P_{0}=0.588\right)$ is surrounded by well-separated side peaks.

$$
\text { 3.2.8. } V 450 \mathrm{Lyr}=\mathrm{KIC} 7671081
$$

The shape of the maxima curve of V450 Lyr suggests a strong beating phenomenon between two modulation periods; however, similar features have also been seen in other stars (e.g., V355 Lyr and KIC 7257008), which proved to be instrumental effects. Accordingly, we carefully compared the frequencies determined in the spectra of the light curve and the $O-C$ diagram. In the case of the light curve, the largest low frequency peak $f_{\mathrm{K}}$ belongs to the Kepler year. The next one is the modulation 

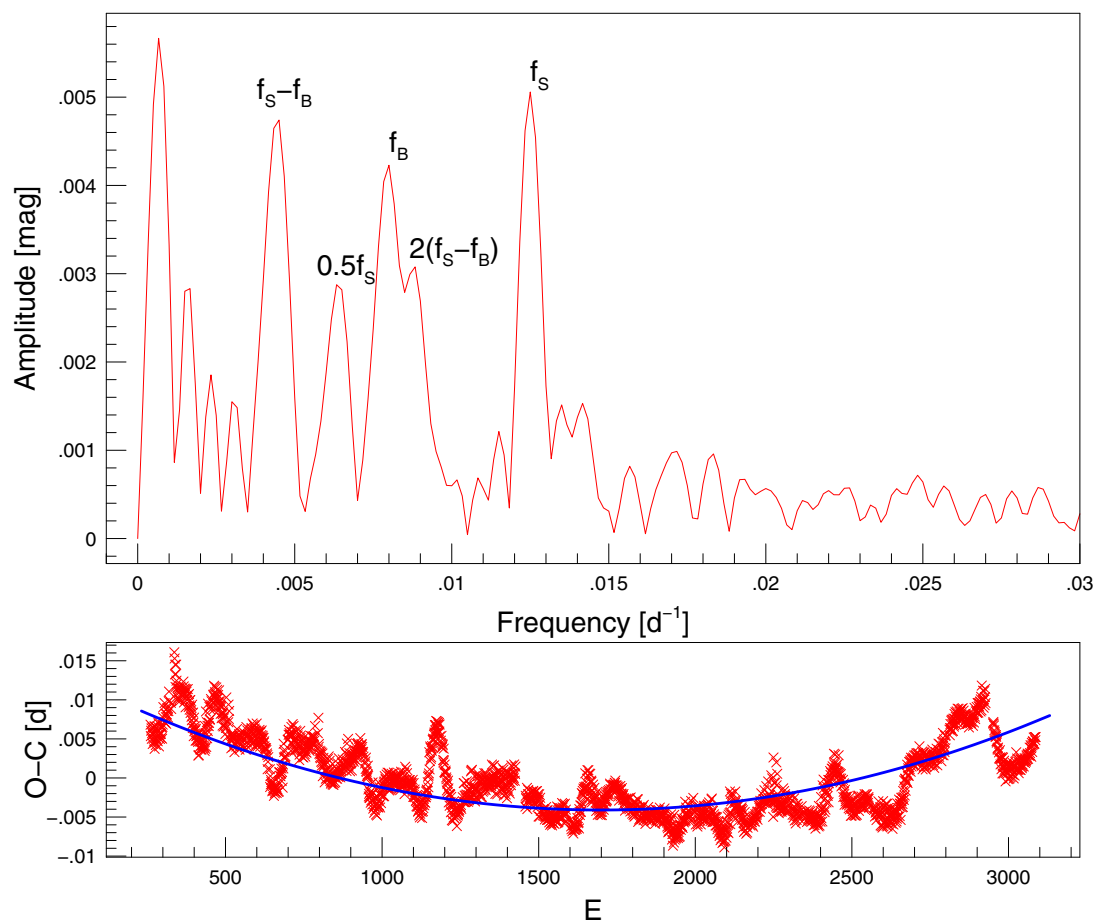

Figure 14. Top: zoom from the Fourier spectrum of the $O-C$ diagram of V450 Lyr. Bottom: residual $O-C$ diagram after we pre-whitened the data with the five frequencies denoted above. The best-fitted parabola (continuous line) suggests a very fast period increase.

(A color version of this figure is available in the online journal.)

frequency $f_{\mathrm{B}}=0.00813 \mathrm{day}^{-1}\left(A\left(f_{\mathrm{B}}\right)=6 \mathrm{mmag}\right)$. Its harmonic $2 f_{\mathrm{B}}$ is also detectable. The third largest amplitude peak is at the frequency $f_{\mathrm{S}}=0.01243 \mathrm{day}^{-1}$. Low significance peaks are seen at the $f_{\mathrm{B}}+f_{\mathrm{S}}$ and $f_{\mathrm{S}} / 2\left(A\left(f_{\mathrm{B}}+f_{\mathrm{S}}\right) \approx A\left(f_{\mathrm{S}} / 2\right) \approx\right.$ 2 mmag).

A similar analysis on the spectrum of $O-C$ diagram (see top panel in Figure 14) results in two independent frequencies, $f_{\mathrm{B}}$ and $f_{\mathrm{S}}$, and some combinations of them $\left(f_{\mathrm{S}}-f_{\mathrm{B}}, f_{\mathrm{S}}-\right.$ $\left.2 f_{\mathrm{B}}, f_{\mathrm{S}} / 2\right)$. We can state now that $f_{\mathrm{S}}$ or $f_{\mathrm{S}} / 2$ is a real secondary modulation frequency (See the discussion about the interpretation of the residual sub-harmonic in Section 3.2.7). When we subtract a fit with all the mentioned frequencies, the residual $O-C$ diagram (bottom panel in Figure 14) shows a combination of the remaining quasi-periodic signal and a parabolic shape indicating a strong period change. Fitting a quadratic function, we found a fast period increase: $d P_{0} / d t=$ $2.4 \times 10^{-8}$ dday $^{-1}$, which cannot be caused by stellar evolution. This phenomenon can be explained, e.g., as a sign of a third longer period modulation or as a random walk caused by a quasi-periodic/chaotic modulation.

By investigating the fine structure of the Fourier spectrum between the harmonics of the main pulsation frequency, Molnár et al. (2014) recognized that V450 Lyr pulsates in the second radial overtone mode as well (see also Figure 6). If we identify the highest peak in this region at 3.33670 day $^{-1}$ with $f_{2}$, the period ratio is $P_{2} / P_{0}=0.594$, corresponding to the canonical second overtone period ratio.

\subsection{9. $V 353 \mathrm{Ly} r=\mathrm{KIC} 9001926$}

The AM of V353 Lyr displays alternating higher and lower amplitude Blazhko cycles (Figure 4). The phenomenon reminds us of the PD effect where the amplitudes of the consecutive pulsation cycles alternate. The effect suggests that the two modulation frequencies have a ratio of nearly 1:2. The low frequency region of the Fourier spectrum of the data is dominated by instrumental frequencies such as $f_{\mathrm{K}}, f_{\mathrm{K}} / 2, f_{\mathrm{Q}}$, and $f_{\mathrm{Q}} / 2$. If we remove these instrumental peaks, we find the two largest noninstrumental ones: $f_{\mathrm{B}}=0.01386 \mathrm{day}^{-1}$ and $f^{(1)}=0.00819 \mathrm{day}^{-1}$. We obtain 0.01394 and $0.00751 \mathrm{day}^{-1}$, respectively, for these values from the spacings of the side peaks. The two frequencies are in the ratio of $\approx 1: 2$ as we predicted. Since we detected the sub-harmonic of the Blazhko frequency for several stars, $f^{(1)}$ could also be a sub-harmonic $\left(f^{(1)}=f_{\mathrm{B}} / 2\right)$ of $f_{\mathrm{B}}$.

The $O-C$ diagram shows alternating maxima and minima again (Figure 7). The Fourier spectrum of this curve is much simpler than the spectrum of the original light curve data. It contains $f_{\mathrm{B}}=0.01395 \mathrm{day}^{-1}$ and its harmonic $2 f_{\mathrm{B}}, f^{(1)}=$ $f_{\mathrm{S}}=0.00761 \mathrm{day}^{-1}$, and an additional discrete peak close to 0 representing a global long-term period change. Removing these three frequencies, we obtain a residual spectrum that contains two significant peaks at $0.01462 \mathrm{day}^{-1}(\mathrm{~S} / \mathrm{N}=11.8)$ and $0.02010 \mathrm{day}^{-1}(\mathrm{~S} / \mathrm{N}=4)$. The most plausible identification of these peaks are $2 f_{\mathrm{S}}$ and $f_{\mathrm{B}}+f_{\mathrm{S}}$, respectively. These frequencies, especially the linear combination, contradict the sub-harmonic scenario.

By removing all the mentioned frequencies, we receive a parabolic shape diagram (which can be seen in Figure 7). The period decrease calculated from the best-fitted parabola is $-8.4 \times 10^{-9}$ dday $^{-1}$ that cannot be explained by an evolutionary effect. It shows hidden period(s) and/or chaotic variation.

To search for additional frequencies, we pre-whitened the data with the main frequency, its harmonics, and the largest 7-8 side peaks around the harmonics. We have found neither the PD effect nor any higher order radial overtone modes (Figure 6).

\subsubsection{V366 Lyr $=$ KIC 9578833}

The maxima of the light curve show a beating-like phenomenon (Figure 4). Calculations from the multiplet structures 


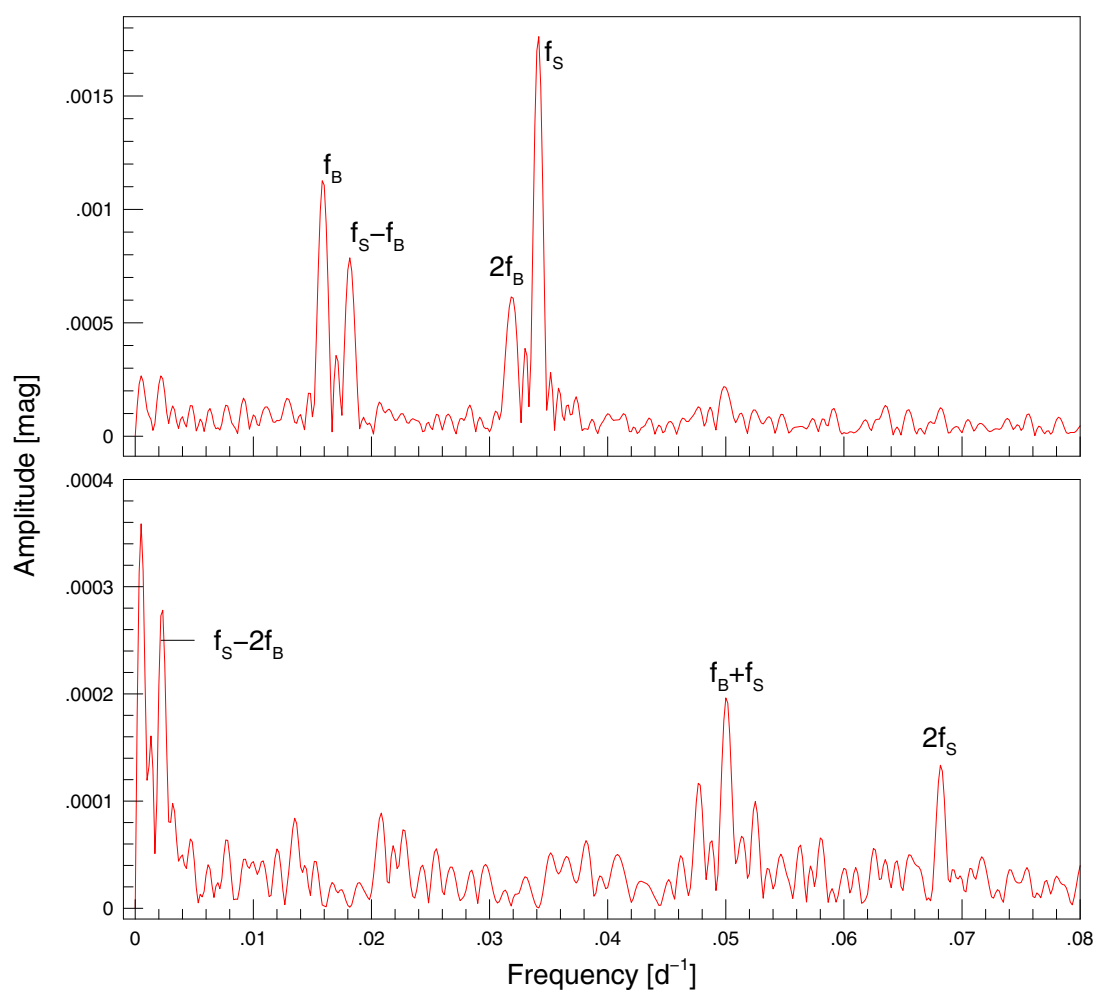

Figure 15. Fourier spectrum of the $O-C$ diagram of V366 Lyr. Top panel: a possible identification of the peaks. Bottom panel: spectrum after we pre-whitened with the four most significant frequencies above.

(A color version of this figure is available in the online journal.)

and the determination of the significant low frequencies end in the same results. Besides the primary modulation frequency $f_{\mathrm{B}}=0.0159 \mathrm{day}^{-1}$, two additional peaks can be detected at the frequencies $f^{(1)}=0.03415$ and $f^{(2)}=0.03175$ day $^{-1}$ with nearly equal amplitudes. The latter is the first harmonic of the Blazhko frequency, but the first one seems to belong to a secondary modulation frequency. Both of the primary $\left(k f_{0} \pm f_{\mathrm{B}}\right)$ and secondary modulation side peaks $\left(k f_{0} \pm f_{\mathrm{S}}\right)$ can be detected. The ratio between the two modulation frequencies is close to $1: 2$. The $O-C$ diagram (in Figure 7) shows a typical beating signal. The spectrum is surprising (Figure 15). Following our expectations, it contains two close frequencies at $f_{\mathrm{B}}=0.015932 \mathrm{day}^{-1}$ and $f^{(3)}=0.01823 \mathrm{day}^{-1}$, which must be responsible for the beating phenomenon. Two other peaks are also visible. One of them is the harmonic of the primary modulation frequency $\left(2 f_{\mathrm{B}}\right)$, while the other is at the position of $f^{(1)}=f_{\mathrm{S}}=0.03414 \mathrm{day}^{-1}$. In this framework, we can identify $f^{(3)}=f_{\mathrm{S}}-f_{\mathrm{B}}$. Strangely enough, the amplitude of $f_{\mathrm{S}}$ is 1.55 times higher (1.7 versus 2.6 minutes) than that of $f_{\mathrm{B}}$. In other words, the roles of the primary and secondary Blazhko modulations are reversed. This is the second case for this new phenomenon (see also V355 Lyr in Section 3.2.7).

There is an alternate identification scenario for the detected frequencies. If we assume $f^{(3)}$ to be the genuine secondary modulation, then the amplitudes would be in order: $A\left(f_{\mathrm{B}}\right)=$ $1.7>A\left(f_{\mathrm{S}}\right)=1.1$ minutes. However, $f^{(1)}$ should be identified as $f_{\mathrm{B}}+f_{\mathrm{S}}$. In this case, (1) the amplitude of the linear combination frequency would be higher than any of its elements. Moreover, since $f^{(1)}$ can be detected directly from the spectrum of the light curve, (2) V366 Lyr would be the only case where a linear combination frequency is detectable instead of the secondary modulation frequency. For the above two (1 and 2) reasons, we prefer the first scenario.

When we pre-whiten the $O-C$ spectrum with the discussed four frequencies, additional significant peaks appear at some harmonics and linear combination frequencies, namely, at $2 f_{\mathrm{S}}$, $f_{\mathrm{S}}+f_{\mathrm{B}}$ and $2 f_{\mathrm{B}}-k f_{\mathrm{S}}$ (bottom panel in Figure 15). After subtracting all these significant frequencies, the residual $O-C$ diagram shows no more structures.

On the basis of Q1-Q2 data, no additional frequency pattern has been found for V366 Lyr (Benkô et al. 2010). The situation is changed when we take into account the Q1-Q16 time span. The highest peak between the harmonics is at the frequency $f^{(4)}=2.675799 \mathrm{day}^{-1}(\mathrm{~S} / \mathrm{N}=4.7$, Figure 6). The period ratio is $P^{(4)} / P_{0}=0.71$. Frequencies with similar period ratios were discovered in the CoRoT targets V1127 Aql and CoRoT 105288363 and the Kepler stars V445 Lyr and V360 Lyr (Chadid et al. 2010; Guggenberger et al. 2012; Benkő et al. 2010). These frequencies are the dominant additional frequencies for only three stars: V1127 Aql, V360 Lyr, and V366 Lyr.

The referred papers generally explain these frequencies as excitation of independent non-radial modes. As we showed in Benkő \& Szabó (2014), all of these frequencies can be constructed by a linear combination as $f^{(4)}=2\left(f_{0}-f_{2}\right)$. Here, in the case of V366 Lyr, we may identify the second overtone mode frequency with the marginal peak at $f_{2}=3.227711$ day $^{-1}$ $(\mathrm{S} / \mathrm{N}=2.7)$. This formal mathematical description has its own strengths and weaknesses. As opposed to the non-radial explanation, it could be verified or denied by using existing radial hydrodynamic codes. It is hardly understandable, however, why the linear combination $2\left(f_{0}-f_{2}\right)$ is stronger than $f_{0}-f_{2}$. 


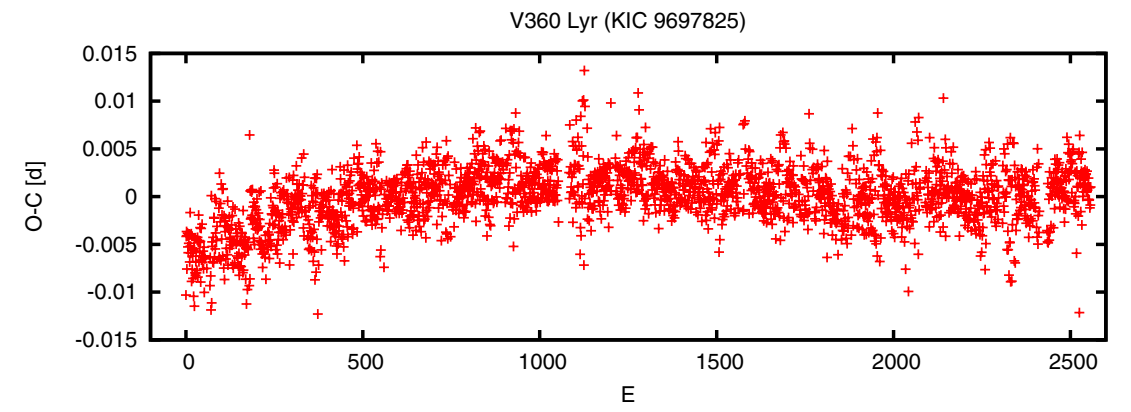

Figure 16. Residual $O-C$ diagram of $\mathrm{V} 360 \mathrm{Lyr}$ after we pre-whitened the data with all significant frequencies.

(A color version of this figure is available in the online journal.)

\subsubsection{V360 Lyr $=$ KIC 9697825}

The maxima of the light curve of V360 Lyr in Figure 4 show a slight beating phenomenon. The Fourier spectrum contains rich multiplet structures around the harmonics of the main pulsation frequency. The largest side peaks indicate the following frequencies: $f_{\mathrm{B}}=0.01919, f^{(1)}=0.02374$, $f^{(2)}=0.02821$, and $f^{(3)}=0.04753$ day $^{-1}$. Two of these frequencies $\left(f_{\mathrm{B}}\right.$ and $\left.f^{(3)}\right)$ can be detected directly as well. If we assume $f^{(3)}$ to be a second independent modulation frequency $\left(f^{(3)}=f_{\mathrm{S}}\right.$ ), the other two values can be expressed as $f^{(2)}=f_{\mathrm{S}}-f_{\mathrm{B}}$ and $f^{(1)}=f_{\mathrm{S}} / 2$.

Beyond the above mentioned four frequencies, the Fourier spectrum of the $O-C$ diagram shows two additional peaks at $2 f_{\mathrm{B}}$ and $f_{\mathrm{S}}+f_{\mathrm{B}}$. We found again a Blazhko star that shows not only a secondary modulation but also its half (see also discussion in Section 3.2.7). The ratio between the two modulation frequencies is 0.404 or 2:5. The residual curve of the $O-C$ diagram (Figure 16) shows a rather long period oscillation as a secular period change.

Two additional frequencies $\left(f_{1}=2.4875\right.$ and $f^{\prime}=$ $2.6395 \mathrm{day}^{-1}$ ) of V360 Lyr were reported by Benkô et al. (2010), who explained $f_{1}$ with a possible first overtone mode and $f^{\prime}$ with an independent non-radial mode. As Szabó et al. (2010) have already mentioned, $f^{\prime}$ could also be a member of a PD pattern around $f^{\prime}=1.5 f_{0}$. When we analyze the Q1-Q16 data set, the highest amplitude peak in this region is located at $f^{(4)}=2.678669$ day $^{-1}\left(P^{(4)} / P_{0}=1.49\right)$, which is undoubtedly a PD frequency.

The interpretation of the strongest additional peak at the frequency of $f^{(5)}=2.487740$ day $^{-1}$ is more problematic. The period ratio $P^{(5)} / P_{0}=0.721$ is far from the canonical value of the first radial overtone and fundamental modes $(0.745)$. Such a ratio could be produced by a highly metal abundant RR Lyrae star (see, e.g., Figure 8 in Chadid et al. 2010), but the metallicity of V360 Lyr is $[\mathrm{Fe} / \mathrm{H}]=-1.5 \pm 0.35 \mathrm{dex}(\mathrm{N} 13)$. This difference might also be due to the resonance, which excites this mode in nontraditional way (Molnár et al. 2012b). We suggested in Benkô \& Szabó (2014) another explanation: similar to V366 Lyr (and V445 Lyr), this frequency could be a linear combination $2\left(f_{2}-f_{0}\right)$, where $f_{2}=3.036015 \mathrm{day}^{-1}$ is the frequency of the second radial overtone mode.

\subsubsection{KIC 9973633}

The history of this star is the same as that of KIC 7257008: the ASAS survey discovered it and its basic parameters were determined for the first time by N13. The Kepler data set of KIC 9973633 is relatively the most unfortunate in the analyzed sample. It has a short time coverage (data exist from only Q10), and two additional quarters (Q11 and Q15) are missing (Figure 4).

Although the triplet structures around the harmonics provide us the Blazhko frequency $f_{\mathrm{B}}=0.01490$ day $^{-1}$, we cannot detect it directly in the low frequency range of the Fourier spectrum. Here the instrumental peak $f_{\mathrm{K}}$ dominates. When we remove it, we find two high amplitude peaks at $f_{\mathrm{K}} / 2$ and $f^{(1)}=0.00411 \mathrm{~d}^{-1}=f_{\mathrm{B}}-f_{\mathrm{Q}}$. After the next pre-whitening step, we can see three peaks near the detection threshold at $f_{\mathrm{Q}}, f^{(2)}=0.03771 \mathrm{day}^{-1}$, and $f^{(3)}=0.02701 \mathrm{day}^{-1}$. It is easily recognizable that $f^{(3)}=f^{(2)}-f_{\mathrm{Q}}$. Which one is the real independent frequency: $f^{(2)}$ or $f^{(3)}$ ?

To investigate this question, we analyzed the $O-C$ diagram of KIC 9973633. The Fourier spectrum shows two highly significant peaks at $f_{\mathrm{B}}=0.01486$ day $^{-1}$ and $2 f_{\mathrm{B}}=0.02972$ day $^{-1}$ (Figure 8). By pre-whitening the data with these two frequencies, we obtain a third one at $0.03675 \mathrm{day}^{-1}$. If we identify it with $f^{(3)}=f_{\mathrm{S}}$, we are in a similar situation to the case of V360 Lyr (Section 3.2.11): we have two modulation frequencies with a ratio of 2:5. The residual of the $O-C$ diagram does not show any structures: it is a constant line with some scatter.

\subsubsection{3. $V 838$ Cyg $=$ KIC 10789273}

The extremely low amplitude Blazhko modulation of V838 Cyg was discovered by N13. The light curve in Figure 4 shows wavy maxima and minima; however, this structure produced by interference of the sampling and the pulsation frequencies does not indicate any AM. It is a virtual modulation and not a real one. It means that we need more careful investigations to decide whether V838 Cyg is modulated or not. Clear triplets around the main pulsation frequency and its harmonics in the Fourier spectrum suggest a modulation phenomenon with the frequency of $f_{\mathrm{B}}=0.01681 \mathrm{day}^{-1}$. It follows that the period is $P_{\mathrm{B}}=59.5$ days, which roughly agrees with the dominant modulation period value (54-55 days) found by N13.

V838 Cyg was observed in SC mode in only one quarter in Q10. We processed these pixel data in the same way as we have done for LC data. The obtained SC light curve does not show virtual modulation like LC data, but shows a small amplitude change. This variation is, however, hard to distinguish from possible instrumental trends. To test the presence of the modulation, we made synthetic data. We prepared an artificial time series $m_{\mathrm{syn}}(t)$ using the Fourier parameters of the observed data such as $f_{0}, A_{k}$ and $\varphi_{k}$, where $A_{k}$ and $\varphi_{k}$ are the amplitude and phase values of the $k$ th harmonics $(k=1,2, \ldots, 11)$, i.e., 

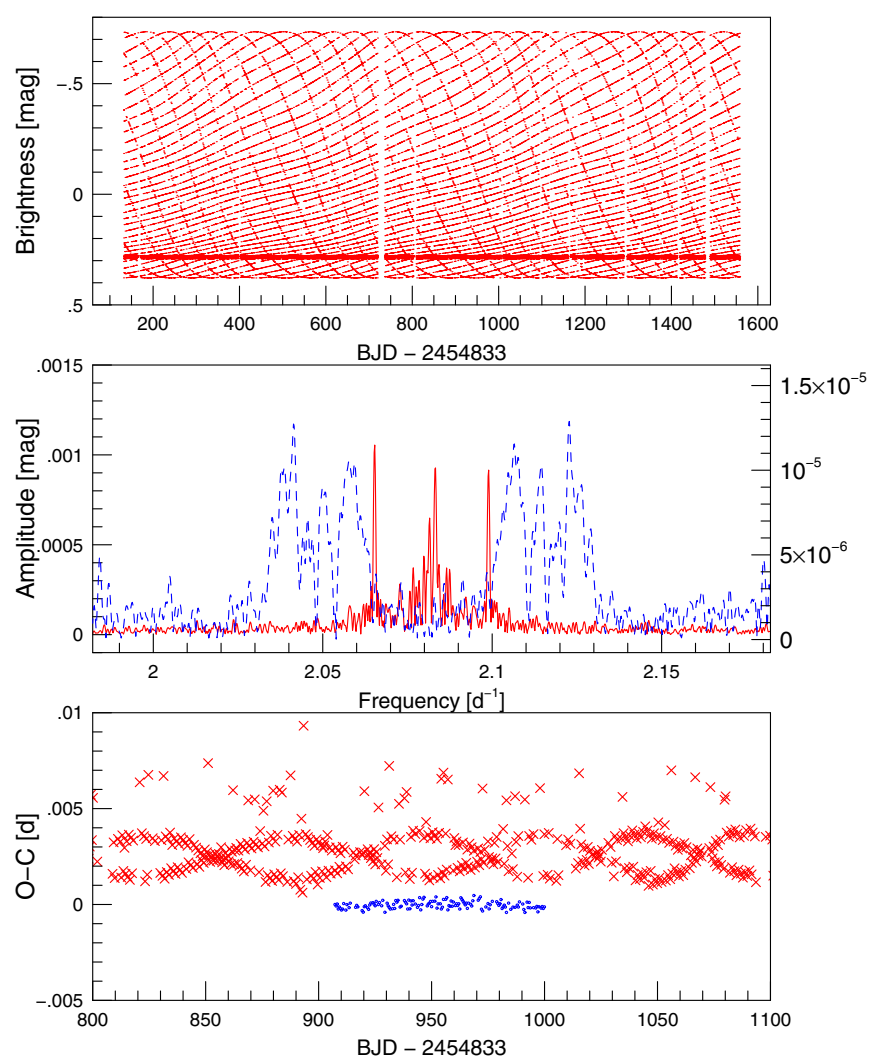

Figure 17. Analysis of V838 Cyg. Top panel: synthetic light curve prepared by using the Fourier parameters of the observed data without assuming any modulations and sampled on the Kepler data points (see Figure 4). Middle panel: Fourier spectra of the observed (red continuous line) and synthetic (blue dashed line) time series around the pulsation frequency after the data were pre-whitened with the main frequency. The left- and right-hand side magnitude scales belong to the observed and synthetic spectra, respectively. Bottom panel: part of the $O-C$ diagrams for $\mathrm{LC}$ data (red crosses) and SC observations (blue dots).

(A color version of this figure is available in the online journal.)

without modulation side peaks:

$$
m_{\text {syn }}(t)=\sum_{k=1}^{11} A_{k} \sin \left(2 \pi f_{0} t+\varphi_{k}\right) .
$$

The synthetic data were sampled at the observed time stamps $t$ (top panel in Figure 17).

The spectra of the observed and synthetic data have systematic differences (see middle panel in Figure 17). (1) In the case of synthetic data, the side peaks have $\approx 80$ times smaller amplitudes than the observed ones $(0.012 \mathrm{mmag}$ versus 1 mmag). (2) The position of the side frequencies are different. (3) The structures of these side patterns are also different: the observed data show simple clear triplets, while the synthetic data produce complicated multiplets. To summarize, we confirmed the AM with an independent method. Since the amplitude of the modulation is very small, we could not detect $f_{\mathrm{B}}$ directly from the low frequency range of the Fourier spectrum. This part of the spectrum is dominated by instrumental frequencies $\left(f_{\mathrm{K}}, f_{\mathrm{L}}\right.$, where $P_{\mathrm{L}}=1 / f_{\mathrm{L}}$ indicates the total length of the observation: $\sim 4$ yr). N13 mentioned possible multiple modulations. After pre-whitening the data with the side frequencies $k f_{0} \pm f_{\mathrm{B}}$, only instrumental side peaks $\left(k f_{0} \pm f_{\mathrm{K}} k f_{0} \pm f_{\mathrm{L}}\right)$ remain significant around the harmonics.
We cannot construct the $O-C$ diagram the traditional way because the sparse continuous sampling with the relatively short pulsation period and the interpolation errors produce systematic undulations (in the bottom panel in Figure 17). Due to the long Blazhko period, the analysis of SC data does not help too much. The sensitive method used by $\mathrm{N} 13$ resulted in 0.001 radian phase variation in $\varphi_{1}$, which predicts about $5 \times 10^{-4} \approx 40 \mathrm{~s} O-C$ variation. It is about the same as our detection limit: the standard deviation of $O-C$ for SC data is $\approx 2 \times 10^{-4}$. Our analysis supports the phase modulation reported by the discovery paper.

We also found additional frequency patterns for the first time in this star. After we subtracted the significant harmonics of the pulsation frequency and triplets around them, the following additional frequencies can be detected between $f_{0}$ and $2 f_{0}$ : $f_{2}=3.509142 \mathrm{day}^{-1}, 1.5 f_{0}=3.113906 \mathrm{day}^{-1}(\mathrm{PD})$, and $3 f_{0}-f_{2}=2.737256$ day $^{-1}$, respectively (see Figure 6).

\subsubsection{KIC 11125706}

Though KIC 11125706 shows the second lowest amplitude AM in our sample, its long pulsation period allows us to detect it without any problems. The asymmetric triplet structures around the pulsation frequency and its harmonics provide $f_{\mathrm{B}}$. Direct detection of this frequency has failed. The low frequency range of the Fourier spectrum is dominated by instrumental peaks.

Contrary to this, the Fourier spectrum of the $O-C$ diagram (Figure 8) contains a very significant $(\mathrm{S} / \mathrm{N}=37.8)$ peak at $f_{\mathrm{B}}$. When we pre-whiten with this frequency, a lower amplitude, but clearly detectable $\left(A\left(f^{(1)}\right)=1.9 \times 10^{-4} \mathrm{~d}, \mathrm{~S} / \mathrm{N}=6.4\right)$, peak can be seen at $f^{(1)}=0.01698 \mathrm{day}^{-1}$. Is it a real secondary modulation frequency or results from the sampling? We generated synthetic $O-C$ data using the Fourier parameters of the primary Blazhko period and added Gaussian noise to it. The synthetic diagram was sampled exactly at the same points as the observed one. The Fourier spectrum of the artificial $O-C$ curve contains only the $f_{\mathrm{B}}$, and it does not show any other significant peaks. Thus, we ruled out an instrumental origin of this frequency. Now, we tend to identify $f^{(1)}=f_{\mathrm{S}}$. In this case the two modulation frequencies have a ratio of nearly $3: 2$. The quadratic fit of the residual $O-C$ gives a slight period increase: $d P_{0} / d t=4.4 \times 10^{-10} \pm 1.1 \times 10^{-10}$ dday $^{-1}$.

Returning to the light curve analysis, we do not find any significant frequencies around $f_{\mathrm{S}}$ and no side peaks detected that could belong to this frequency. These facts mean that the AM of $f_{\mathrm{S}}$ is below our detection limit. Furthermore, no additional peak patterns have been found between harmonics (Figure 6).

$$
\text { 3.2.15. V1104 Cyg }=\text { KIC } 12155928
$$

Beyond V2178 Cyg, V1104 Cyg was also the subject of the case study of N13. We can add only few things to this study. We derived the Blazhko cycle based on a longer time span. The frequency of the Blazhko modulation $f_{\mathrm{B}}$ is highly significant both in the Fourier spectrum of the light curve and that of the $O-C$ diagram. The latter spectrum also includes the harmonic $2 f_{\mathrm{B}}$ (Figure 8), indicating non-sinusoidal behavior of the FM. The $O-C$ residual does not indicate any period changes during the Kepler timescale. Our analysis resulted in neither secondary modulation nor additional frequencies between harmonics (Figure 6).

\section{CONCLUSIONS}

The main goal of this paper was to investigate the longtimescale behavior of the Blazhko effect among the Kepler 
RR Lyrae stars. To provide the best input for the analysis, we prepared time series from the pixel photometric data with our own tailor-made aperture. These light curves include the total flux of the stars for only nine cases, while some portion of the flux of six RR Lyrae stars was lost using even in the largest possible aperture. Nevertheless, our data set comprises the longest continuous, most precise observations of Blazhko RR Lyrae stars ever published. These data will be unprecedented for years to come.

Since the Blazhko effect manifests itself in simultaneous AM and FM, we analyzed both phenomena and compared the results of the separated analyses. This approach reduces the influence of the instrumental effects.

We detected single Blazhko period for three stars: V783 Cyg, V838 Cyg, and V1104 Cyg. Since V838 Cyg shows the smallest amplitude modulation both in the AM and the FM, we could not confirm its multiperiodicity that was suspected by N13.

Twelve stars in our sample show evidence for multiperiodic modulation. In eight cases, we could determine two significant Blazhko periods, while for four additional cases (V2178 Cyg, V808 Cyg, V354 Lyr, and KIC 7257008) we could establish the presence of a possible long secondary period. It does not mean, however, that we could describe the total variations with these one or two periods completely. The residual curves show significant structures (see, e.g., V2178 Cyg, V808 Cyg, and V450 Lyr) after subtracting the best-fitted light/ $O-C$ curves. For the two to three stars with the shortest Blazhko periods, we have a chance to carry out a dynamical analysis. In this work, we mentioned the preliminary result from the cycle-tocycle variation of V783 Cyg modulation (E. Plachy et al., in preparation), which hints at its chaotic nature.

The latest and most complete compilation of the Galactic Blazhko RR Lyrae stars (Skarka 2013) consists of only 8 multiperiodic cases among 242 known field Blazhko RR Lyrae stars (3.3\%). More recently, Skarka (2014) studied a more homogeneous 321 element sample from the ASAS and SuperWASP surveys. He found the ratio of the multiperiodic and irregularly modulated stars to be $12 \%$. In this work, we surprisingly found that most of the Kepler Blazhko stars-12 out of 15 $(80 \%)$ - belong to the multiperiodic group. In other words, the Blazhko effect predominantly manifests as a multiperiodic phenomenon instead of a mono-periodic and regular one. Here we briefly summarize the main characteristics of the multiperiodic modulations.

1. Up to now the known smaller amplitude (secondary) modulation periods were generally longer than the primary ones. The only exception is RZ Lyr (Jurcsik et al. 2012). Here we showed five further examples (V355 Lyr, V450 Lyr, V366 Lyr, V360 Lyr, and KIC 9973633) for shorter secondary periods.

2. Also, the definition of the primary and secondary modulations proved to be relative. In three cases (for V450 Lyr, V366 Lyr, and V355 Lyr) the relative strength of the primary modulations is weaker in the FM than in the AM.

3. The linear combination of the modulation frequencies can generally be detected, which indicates the nonlinear coupling between the modes. Sub-harmonic frequencies $\left(f_{\mathrm{B}} / 2\right.$ and/or $\left.f_{\mathrm{S}} / 2\right)$ were detected for numerous cases (KIC 7257008, V355 Lyr, V450 Lyr, and V360 Lyr). In the majority of the cases, the two modulation periods are in a ratio of small integer numbers: 1:2 for V353 Lyr; 2:1 for V366 Lyr and V355 Lyr (3:2 for V2178 Cyg); 2:3 for KIC 11125706; and 5:2 for KIC 9973633 and V360 Lyr. (Here the ratios of the primary AM period versus the secondary one are indicated.)

As a by-product of the analysis, we report here for the first time additional frequency structures for V808 Cyg, V355 Lyr, and V838 Cyg. For all three cases we detected the second radial overtone mode $f_{2}$.

The former studies of CoRoT and Kepler Blazhko data found unidentified frequencies for numerous stars. These frequencies were explained by non-radial mode excitation. Here we showed that almost all of such frequencies can also be produced by linear combinations of radial modes. The only case where we could not find a proper linear combination is the highest amplitude additional frequency of V354 Lyr.

The amplitudes of these frequencies point to the non-radial mode scenario. These non-radial modes may be excited by resonances at the locations of the linear combination of the radial modes (Van Hoolst et al. 1998). In other words, these frequencies are linear combinations from mathematical point of view only, and physically they are frequencies of independent (non-radial) modes.

Funding for this Discovery mission is provided by NASA's Science Mission Directorate. This project has been supported by the "Lendület-2009 Young Researchers" Program of the Hungarian Academy of Sciences, the Hungarian OTKA grant K83790, and the KTIA URKUT_10-1-2011-0019 grant. The research leading to these results has received funding from the European Community's Seventh Framework Programme (FP7/2007-2013) under grant agreement No. 269194 (IRSES/ ASK) and No. 312844 (SPACEINN). The work of E. Plachy was supported by the European Union and the State of Hungary, co-financed by the European Social Fund in the framework of TÁMOP 4.2.4. A/2-11-1-2012-0001 "National Excellence Program.” R. Szabó was supported by the János Bolyai Research Scholarship of the Hungarian Academy of Sciences. The authors thank the referee for carefully reading the manuscript and for helpful suggestions.

\section{REFERENCES}

Bányai, E., Kiss, L. L., Bedding, T., et al. 2013, MNRAS, 436, 1576 Benkő, J. M., Kolenberg, K., Szabó, R., et al. 2010, MNRAS, 409, 1585 Benkő, J. M., \& Szabó, R. 2014, in IAU Symp. 301, Precision Asteroseismology, ed. J. A. Guzik, W. J. Chaplin, G. Handler, \& A. Pigulski (Cambridge: Cambridge Univ. Press), 383

Benkő, J. M., Szabó, R., \& Paparó, M. 2011, MNRAS, 417, 974

Blazhko, S. N. 1907, AN, 175, 325

Breger, M., Handler, G., Garrido, R., et al. 1999, A\&A, 349, 225

Buchler, J. R., \& Kolláth, Z. 2011, ApJ, 731, 24

Chadid, M., Benkő, J. M., Szabó, R., et al. 2010, A\&A, 510, A39

Cross, J. 1991, JAVSO, 20, 214

Fanelli, M. N., Jenkins, J. M., Bryson, S., et al. 2011, Kepler Data Processing Handbook (Moffett Field: NASA Ames Research Center)

Guggenberger, E., Kolenberg, K., Nemec, J. M., et al. 2012, MNRAS, 424, 649

Jenkins, J. M., Caldwell, D. A., Barclay, Th., et al. 2013, Kepler Data Characteristics Handbook (Moffett Field: NASA Ames Research Center)

Jenkins, J. M., Caldwell, D. A., Chandrasekaran, H., et al. 2010a, ApJL, 713, L87

Jenkins, J. M., Caldwell, D. A., Chandrasekaran, H., et al. 2010b, ApJL, 713, L120

Jurcsik, J., Sódor, Á., Hajdu, G., et al. 2012, MNRAS, 427, 1517

Koch, D. G., Borucki, W. J., Basri, G., et al. 2010, ApJL, 713, L79

Kolenberg, K. 2012, JAVSO, 40, 481

Kolenberg, K., Bryson, S., Szabó, R., et al. 2011, MNRAS, 411, 878

Kolenberg, K., Szabó, R., Kurtz, D. W., et al. 2010, ApJL, 713, L198

Kolláth, Z. 1990, Occ. Tech. Notes. Konkoly Obs. No 1

Kolláth, Z., Buchler, J. R., Szabó, R., \& Csubry, Z. 2002, A\&A, 385, 932 
Kolláth, Z., Molnár, L., \& Szabó, R. 2011, MNRAS, 414, 111

Kovács, G. 2009, in AIP Conf. Proc. 1170, Stellar Pulsation: Challanges for Theory and Observation, ed. J. A. Guzik \& P. Bradley (Melville, NY: AIP), 26

Lenz, P., \& Breger, M. 2005, CoAst, 146, 53

Molnár, L., Benkő, J. M., Szabó, R., \& Kolláth, Z. 2014, in IAU Symp. 301, Precision Asteroseismology, ed. J. A. Guzik, W. J. Chaplin, G. Handler, \& A. Pigulski (Cambridge: Cambridge Univ. Press), 459

Molnár, L., Kolláth, Z., \& Szabó, R. 2012a, MNRAS, 424, 31

Molnár, L., Kolláth, Z., Szabó, R., et al. 2012b, ApJL, 757, L13

Nemec, J. M., Cohen, J. G., Ripepi, V., et al. 2013, ApJ, 773, 181 (N13)

Nemec, J. M., Smolec, R., Benkô, J. M., et al. 2011, MNRAS, 417, 1022

Pojmanski, G. 1997, AcA, 47, 467

Pojmanski, G. 2002, AcA, 52, 397
Reegen, P. 2007, A\&A, 467, 1353

Skarka, M. 2013, A\&A, 549, A101

Skarka, M. 2014, A\&A, 562, A90

Smolec, R., Moskalik, P., Kolenberg, K., et al. 2011, MNRAS, 414, 2950

Sódor, Á., Jurcsik, J., Szeidl, B., et al. 2011, MNRAS, 411, 1585

Sterken, C. 2005, in ASP Conf. Ser. 335, The Light-Time Effect in Astrophysics, ed. C. Sterken (San Francisco, CA: ASP), 3

Stothers, R. B. 2006, ApJ, 653, 73

Szabó, R., Benkő, J. M., Paparó, M., et al. 2014, A\&A, submitted

Szabó, R., Kolláth, Z., Molnár, L., et al. 2010, MNRAS, 409, 1244

Van Cleve, J., Caldwell, D., Thompson, R., et al. 2009, Kepler Instrumental Handbook (Moffett Field: NASA Ames Research Center)

Van Hoolst, T., Dziembowski, W. A., \& Kawaler, S. D. 1998, MNRAS, 297,536 